\title{
GALAXY EVOLUTION IN A COMPLEX ENVIRONMENT: A MULTI-WAVELENGTH STUDY OF HCG 7*
}

\author{
I. S. Konstantopoulos ${ }^{1}$, S. C. Gallagher ${ }^{2}$, K. Fedotov $^{2}$, P. R. Durrell ${ }^{3}$, A. Heiderman ${ }^{4}$, D. M. Elmegreen ${ }^{5}$, \\ J. C. Charlton ${ }^{1}$, J. E. Hibbard ${ }^{6}$, P. Tzanavaris ${ }^{7,8}$, R. Chandar ${ }^{9}$, K. E. Johnson ${ }^{6,10}$, A. Maybhate ${ }^{11}$, \\ A. E. ZabludofF ${ }^{12}$, C. Gronwall ${ }^{1}$, D. Szathmary ${ }^{2}$, A. E. Hornschemeier ${ }^{7}$, J. English ${ }^{13}$, B. Whitmore ${ }^{11}$, \\ C. Mendes de Oliveira ${ }^{14}$, and J. S. Mulchaey ${ }^{15}$ \\ ${ }^{1}$ Department of Astronomy and Astrophysics, The Pennsylvania State University, University Park, PA 16802, USA; iraklis@ psu. edu \\ 2 Department of Physics \& Astronomy, The University of Western Ontario, London, ON, N6A 3K7, Canada \\ ${ }^{3}$ Department of Physics \& Astronomy, Youngstown State University, Youngstown, OH 44555, USA \\ ${ }^{4}$ Department of Astronomy, University of Texas at Austin, Austin, TX 78712-0259, USA \\ ${ }^{5}$ Department of Physics \& Astronomy, Vassar College, Poughkeepsie, NY 12604, USA \\ ${ }^{6}$ National Radio Astronomy Observatory, Charlottesville, VA, USA \\ ${ }^{7}$ Laboratory for X-ray Astrophysics, NASA's Goddard Space Flight Center, Greenbelt, MD 20771, USA \\ ${ }^{8}$ Department of Physics and Astronomy, The Johns Hopkins University, Baltimore, MD 21218, USA \\ ${ }^{9}$ University of Toledo, Toledo, OH, USA \\ ${ }^{10}$ University of Virginia, Charlottesville, VA, USA \\ ${ }^{11}$ Space Telescope Science Institute, Baltimore, MD, USA \\ ${ }^{12}$ Steward Observatory, University of Arizona, Tucson, AZ 85721, USA \\ ${ }^{13}$ University of Manitoba, Winnipeg, MN, Canada \\ ${ }^{14}$ Universidade de São Paulo, IAG, São Paulo, SP 05508-900, Brazil \\ ${ }^{15}$ Carnegie Observatories, Pasadena, CA 91101, USA \\ Received 2010 February 26; accepted 2010 July 15; published 2010 October 8
}

\begin{abstract}
The environment where galaxies are found heavily influences their evolution. Close groupings, like the ones in the cores of galaxy clusters or compact groups, evolve in ways far more dramatic than their isolated counterparts. We have conducted a multi-wavelength study of Hickson Compact Group 7 (HCG 7), consisting of four giant galaxies: three spirals and one lenticular. We use Hubble Space Telescope (HST) imaging to identify and characterize the young and old star cluster populations. We find young massive clusters (YMCs) mostly in the three spirals, while the lenticular features a large, unimodal population of globular clusters (GCs) but no detectable clusters with ages less than a few Gyr. The spatial and approximate age distributions of the $\sim 300$ YMCs and $\sim 150$ GCs thus hint at a regular star formation history in the group over a Hubble time. While at first glance the HST data show the galaxies as undisturbed, our deep ground-based, wide-field imaging that extends the HST coverage reveals faint signatures of stellar material in the intragroup medium (IGM). We do not, however, detect the IGM in H I or Chandra X-ray observations, signatures that would be expected to arise from major mergers. Despite this fact, we find that the H I gas content of the individual galaxies and the group as a whole are a third of the expected abundance. The appearance of quiescence is challenged by spectroscopy that reveals an intense ionization continuum in one galaxy nucleus, and post-burst characteristics in another. Our spectroscopic survey of dwarf galaxy members yields a single dwarf elliptical galaxy in an apparent stellar tidal feature. Based on all this information, we suggest an evolutionary scenario for HCG 7, whereby the galaxies convert most of their available gas into stars without the influence of major mergers and ultimately result in a dry merger. As the conditions governing compact groups are reminiscent of galaxies at intermediate redshift, we propose that HCGs are appropriate for studying galaxy evolution at $z \sim 1-2$.
\end{abstract}

Key words: galaxies: evolution - galaxies: groups: individual (HCG 7) - galaxies: interactions - galaxies: star clusters: general - galaxies: star formation

Online-only material: color figures

\section{INTRODUCTION}

The high luminosities and impressive, disturbed morphologies of local merging and interacting galaxies testify to the ability of these processes to drive rapid galaxy evolution. Such events often result in increased star formation activity, ${ }^{16}$ as testified by the enhanced infrared (IR) luminosities (Sanders \& Mirabel 1996), and the redistribution of the gaseous and stellar components of the individual galaxies (Holtzman et al. 1992; Hibbard \& van Gorkom 1996; Schweizer \& Seitzer 1998; Zhang \& Fall 1999; Whitmore et al. 1999; Trancho et al. 2007b;

\footnotetext{
* Based on observations made with the NASA/ESA Hubble Space Telescope. 16 This might not always be the case, however, as challenged by Bergvall et al. (2003), who presented mergers as scaled-up versions of isolated spirals, without a necessary SFR enhancement.
}

Konstantopoulos et al. 2010). Galaxies, however, are also found in isolation. In the absence of external influences, including tidal forces from massive galaxies and interactions with intracluster or intragroup media (IGMs), these true "island universes" can evolve through internal processes such as disk instabilities that boost star formation and funnel gas to smaller radii (e.g., Kormendy \& Kennicutt 2004, and references therein). In cases where internal dynamics dominate external processes over cosmological timescales, we speak of secular (i.e., continuous and cumulative) evolution. The relative importance of these two processes is under debate. Indeed, the latter may have been discounted during the past two decades of Hubble Space Telescope (HST) driven studies of intensely star-forming environments. Under both scenarios, dynamical processes in concert with the conversion of gas into stars can lead to the 
morphological evolution of disk-dominated galaxies from gas rich and star forming to quiescent and featureless with substantial bulge growth. Interestingly, the mechanisms that drive this transformation are far from certain. Do mergers and interactions represent the main path from star-forming spirals to quiescent ellipticals (e.g., Genzel et al. 2001), or does secular evolution represent an equally relevant (at $z \sim 0$ ), albeit less eventful path?

Most galaxies are not found at the extremes of true isolation in the field or conversely within galaxy cluster cores, but in the intermediate density environments of groups (Eke et al. 2004). In such systems, both types of evolutionary mechanisms are likely to be operative; many galaxies will have undergone interactions and mergers, but the dynamical times are long enough that not all galaxies will be affected. Compact galaxy groups, with several galaxies within a few galaxy radii, offer excellent arenas for investigating morphological evolution triggered by interactions at high number density; their low velocity dispersions, $\sigma \sim$ $10^{2} \mathrm{~km} \mathrm{~s}^{-1}$ (Tago et al. 2008), with respect to cluster cores ( $\sim 10^{3} \mathrm{~km} \mathrm{~s}^{-1}$; e.g., Binggeli et al. 1987; The \& White 1986) extend the timescales of gravitational encounters. Furthermore, the IGM in all but the most massive groups is insufficient for ram-pressure stripping, which simplifies the investigation of the agents of morphological change in comparison with clusters (Rasmussen et al. 2008).

Hickson Compact Groups (HCGs; Hickson 1982; Hickson et al. 1992) provide well-studied examples of this fruitful environment. Initially selected based on high number density and isolation (to exclude clusters), they are found to be generally H I deficient when compared to field galaxies of matched morphologies (Williams \& Rood 1987; Verdes-Montenegro et al. 2001), implying that accelerated gas consumption is already underway. HCGs as a population are diverse, and range from conglomerations of small, gas-rich galaxies apparently coming together for the first time such as HCG 31 (e.g., Gallagher et al. 2010; Rubin et al. 1991) to massive, highly evolved and X-ray bright groups such as HCG 42 that are dominated by quiescent ellipticals (e.g., Zabludoff \& Mulchaey 1998). Another interesting trait exhibited by HCGs is a gap in the mid-IR color distribution of member galaxies (Johnson et al. 2007; Walker et al. 2009, hereafter J07 and W09) that is not present in test samples of field galaxies. This can be interpreted as evidence of rapid evolution from gas rich and star forming to gas poor and quiescent, possibly accompanied by the buildup of a bulge. At the same time, the high fraction $(\sim 43 \%)$ of optically evident morphological disturbances in compact group galaxies (Mendes de Oliveira \& Hickson 1994) does not map onto correspondingly high star formation rates (SFRs) from infrared surveys (Moles et al. 1994; Verdes-Montenegro et al. 1998). In some sense, the dynamical state (i.e., the strength of morphological interaction indicators) of a compact group is not a good predictor of its ongoing star formation activity (Iglesias-Páramo \& Vílchez 1999). Therefore, the history of direct collisions does not provide the whole story.

\subsection{An Evolutionary Sequence for Compact Galaxy Groups}

Our previous work has dealt with compact groups both individually (Gallagher et al. 2010) and in the ensemble (J07; W09; Gallagher et al. 2008; Tzanavaris et al. 2010). Our sample is composed of the 12 groups in the Hickson catalog within $4500 \mathrm{~km} \mathrm{~s}^{-1}$ with at least three accordant members that are compact enough to fit within a few arcminutes (for efficient observing). To investigate the evolutionary stage of individual groups as a whole, J07 classified them as early, intermediate, or late following the qualitative scheme of Verdes-Montenegro et al. (2001) according to their neutral gas content (from rich to poor) and morphological state (from spiral-dominated to E/SOdominated) of the galaxies. In tandem, groups were divided quantitatively into three categories according to the total gas available to the system divided by the dynamical mass, as presented in Figure 1. This ratio of gas-to-dynamical mass may serve as a proxy of evolutionary stage, though the mapping is not one to one. J07 classified three categories: (I) relatively H Irich, $\frac{\log \left(M_{\mathrm{HI}}\right)}{\log \left(M_{\mathrm{dyn}}\right)} \geqslant 0.9$; (II) intermediate H I, $0.9>\frac{\log \left(M_{\mathrm{HI}}\right)}{\log \left(M_{\mathrm{dyn}}\right)} \geqslant 0.8$; and (III) relatively $\mathrm{H}$ I-poor, $\frac{\log \left(M_{\mathrm{HI}}\right)}{\log \left(M_{\mathrm{dyn}}\right)}<0.8$.

\subsection{Lenticular Galaxies in the Group Environment}

From their recent comparison of $z \sim 0.4$ field and group galaxies, Wilman et al. (2009) conclude that the high S0 fraction in galaxy clusters is driven by infalling groups, but the lenticulars are forged through preprocessing in the group environment. Some intriguing results from local compact group studies suggest that this environment may offer fertile ground for exploring this issue. For example, a kinematic study of HCG 90 by Plana et al. (1998) revealed that two member galaxies, HCG 90b and d, morphologically classified as ellipticals, have rotating stellar disks and are more accurately classified as S0s. This group is also notable for its large fraction ( 38\%-48\%) of intracluster light compared to total group light and very faint X-ray-emitting IGM (White et al. 2003). Similarly, Seyfert's Sextet (HCG 79), obviously undergoing intense and complex interactions from its highly distorted member galaxies, revealed a surprising lack of young star clusters (Palma et al. 2002) compared to the similarly disrupted Stephan's Quintet (HCG 92; Gallagher et al. 2001). In this paper, we turn to HCG 7 to flesh out the intermediate stages of one type of compact group evolution that plausibly results in systems such as HCG 79 and 90.

\section{3. $H C G 7$}

Group 7 in the Hickson catalog consists of four galaxies: three barred spirals, NGC 192, NGC 201, and NGC 197 (members A, C, and D), and one barred lenticular, NGC 196 (B). We will refer to the galaxies by their alphabetical classifiers, $A, B, C$, and $D$ throughout this paper. We present new HST imaging of the group in two pointings in Figure 2. $A$ is highly inclined, while $D$ projects a rather limited extent and faint, asymmetric spiral structure. The imposing $C$ displays a clear, face-on view of its two dominant spiral arms, one of which may have a composite structure. Positional, morphological, photometric, and redshift information has been included in Table 1 .

Perhaps most interestingly, all spirals in this group appear to be undisturbed, thus HCG 7 offers the opportunity for an excellent case study of galaxy evolution in the group environment in the absence of obvious strong interactions. This work forms part of a series of papers that use HCGs as probes of galaxy evolution in dense environments (J07; W09; Gallagher et al. 2008, 2010; Tzanavaris et al. 2010). Specifically, we are applying an extensive, multi-wavelength data set of a sample of 12 nearby HCGs to the problem of characterizing star formation and following gas consumption and distribution within compact galaxy groups.

Over the following sections, we present an in-depth study of the group, assess its current state, interpret its past, and 


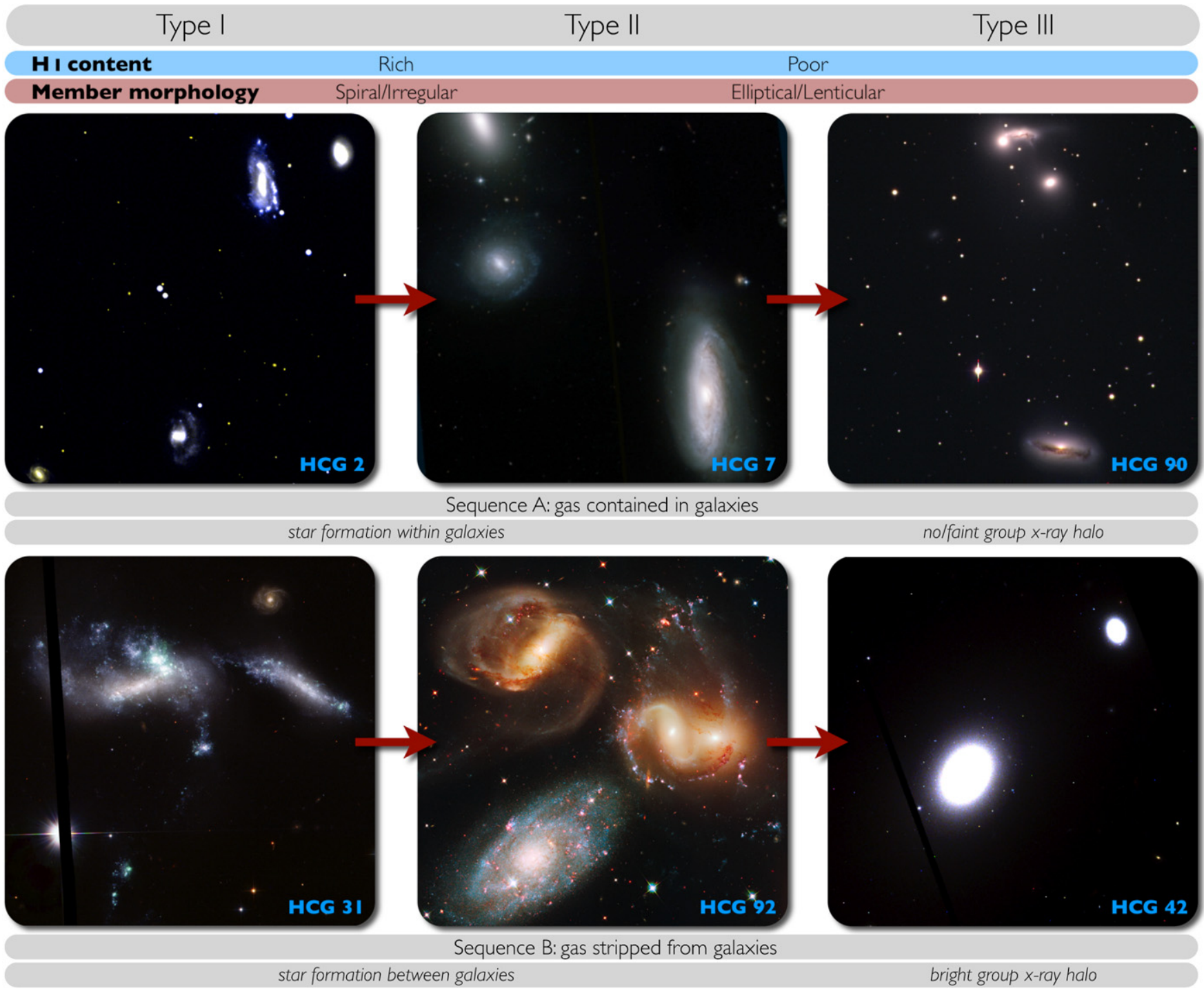

Figure 1. Proposed double-branched evolutionary sequence for compact galaxy groups. The upper sequence shows the evolution of groups in the absence of gas in the IGM. Under this scenario, star formation proceeds uninterrupted in the individual galaxies and the HI gas is consumed before major interactions can take place within the group. Thus, gas does not play a significant role in the ensuing "dry merger." The lower sequence shows a better studied situation, where a number of strong interactions take place at early times in the course of the group's evolution. In that way, the IGM is enriched with gas that is used partly in forming stars, while another part contributes to the X-ray IGM. Our measurements of the way in which gas is processed in HCGs may be related to the $L_{X} / L_{B}$ observed in the current-era universe. (A color version of this figure is available in the online journal.)

attempt to predict its evolution. We will provide the first highresolution study of the young stellar populations and cluster complexes in HCG 7, and the first study of globular clusters (GCs) in a compact group lenticular. This will then be put in the context of galaxy evolution by comparison to field galaxies and other compact groups. The following two sections (Sections 2 and 3) present the optical and infrared imaging, radio data, and optical spectroscopy we have used to dissect this intriguing system. Section 4 includes the analysis of the stellar content at all levels of the star formation hierarchy, i.e., young and old star clusters, cluster-forming regions/complexes, and dwarf galaxies. The GC population is treated separately in Section 5. An interpretation of the multi-wavelength properties of each group galaxy is presented in Section 6. We discuss the implications of our findings in Section 7, where we also explore the significance of HCG 7 in the context of compact group evolution. We summarize our findings in Section 8. The analysis presented in this paper assumes the set of cosmological parameters of Spergel et al. (2007): $H_{0}=0.70, \Omega_{m}=0.3$, and $\Omega_{\lambda}=0.7$.

\section{HST ACS IMAGING AND CLUSTER CANDIDATE SELECTION}

The basis of our analysis of HCG 7 is the HST multiband imaging, obtained using the Wide Field Channel (WFC) of the Advanced Camera for Surveys (ACS), in the F435W, $F 606 \mathrm{~W}$, and $F 814 \mathrm{~W}$ bands and in two pointings (to cover all group members). We adopt the notation $B_{435}, V_{606}$, and $I_{814}$ to relate the observations to the Johnson photometric system, however, the notation does not imply a conversion between the two systems. The observations were executed on 2006 September 10 and 11, as part of program 10787 (PI: J. Charlton). The total exposure times were 1710, 1230, and $1065 \mathrm{~s}$ in the $B V I$ bands, respectively. The observations for each filter were taken with three equal exposures, using a 

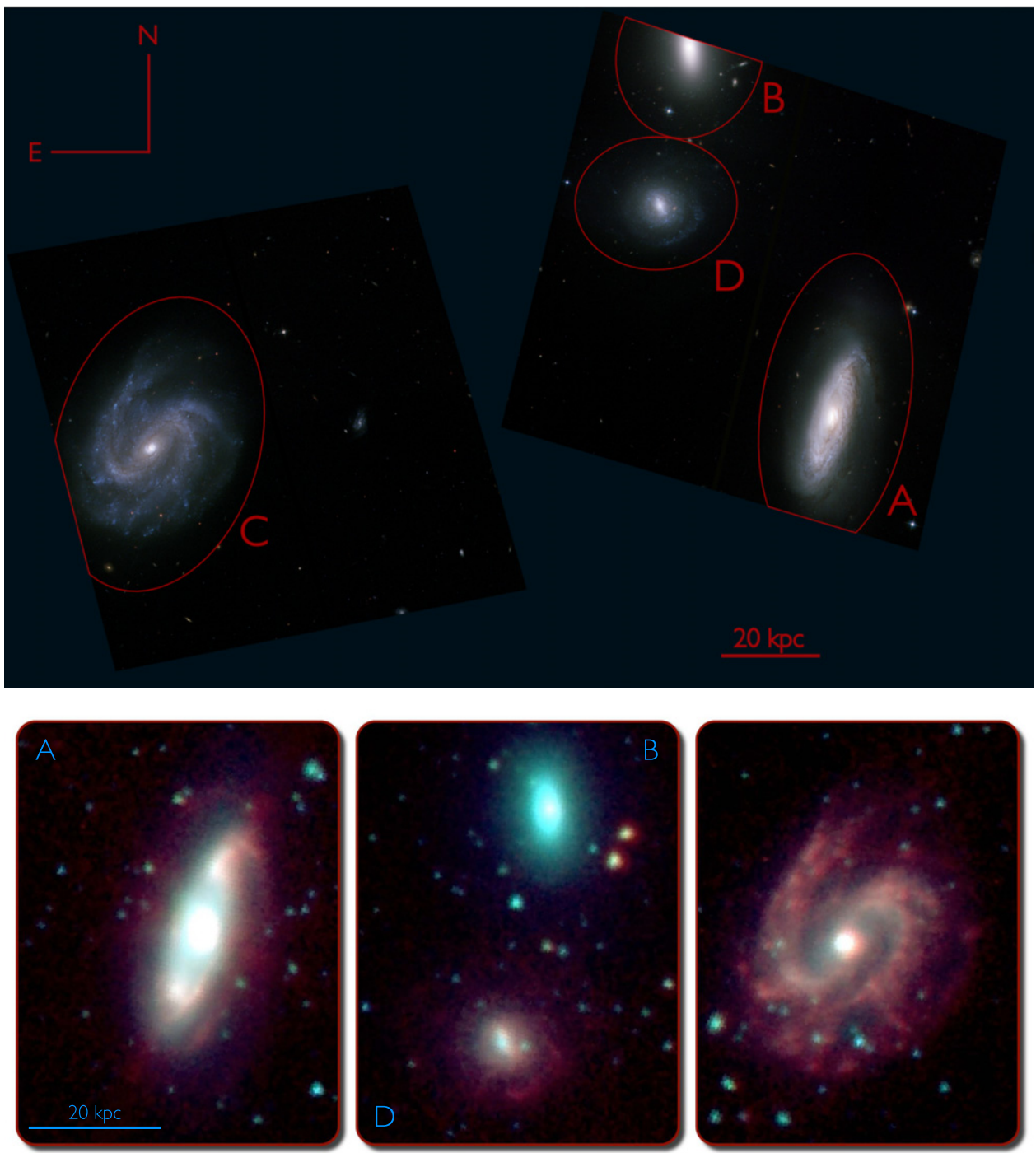

Figure 2. Top: HST BVI color composite image of the four galaxies in two pointings (see Table 1 for basic information). The image covers all four group member galaxies. $A, C$, and $D$ (NGC 192, 201, and 197, respectively) are barred spiral galaxies; $A$ is highly inclined, while $D$ projects a rather limited extent and very faint spiral structure. $B$ (NGC 196) is a barred lenticular galaxy (SB0). The ellipses indicate the regions we have associated to each of the galaxies; any objects found outside those areas are considered to occupy the intracluster medium. From the optical image alone, it is obvious that the four galaxies are "strangers," in the sense that they show no record of past interactions. Bottom: Spitzer imaging, color-coded with blue, green, and red colors corresponding to 3.6, 4.5, and $8 \mu \mathrm{m}$. In that way, photospheric emission from the old stellar population shines blue, while star-forming regions are revealed by the bright red PAH emission.

(A color version of this figure is available in the online journal.)

Table 1

Basic Information on HCG 7 Member Galaxies ${ }^{\mathrm{a}}$

\begin{tabular}{|c|c|c|c|c|c|}
\hline \multirow[t]{2}{*}{ Identifier } & \multirow{2}{*}{$\begin{array}{c}\text { Coordinates } \\
\text { (J2000) }\end{array}$} & \multicolumn{2}{|c|}{ Type } & \multirow{2}{*}{$\begin{array}{c}m^{\mathrm{a}} \\
(\mathrm{mag})\end{array}$} & \multirow{2}{*}{$\begin{array}{c}v_{R}^{\mathrm{b}} \\
\left(\mathrm{km} \mathrm{s}^{-1}\right)\end{array}$} \\
\hline & & $\mathrm{H} 89^{\mathrm{c}}$ & $\mathrm{RC} 3$ & & \\
\hline A: NGC 192 & $003913.4+005151$ & SB & (RÕ)SB(r)a & $13.4(V)$ & 4160 \\
\hline B: NGC 196 & $003917.8+005446$ & SB0 & SB0pec & $13.8(V)$ & 4135 \\
\hline C: NGC 201 & $003934.8+005136$ & $\mathrm{SBc}$ & $\mathrm{SAB}(\mathrm{r}) \mathrm{c}$ & $13.8(g)$ & 4356 \\
\hline D: NGC 197 & $003918.8+005331$ & $\mathrm{SBc}$ & SB0pec & $15.4(g)$ & 4107 \\
\hline E: - & $003915.5+005633$ & - & $\mathrm{dE}^{\mathrm{d}}$ & 18.5 & 4118 \\
\hline
\end{tabular}

Notes.

${ }^{a}$ From the Third Reference Catalogue of Bright Galaxies, de Vaucouleurs et al. (1991, RC3).

${ }^{\mathrm{b}}$ Measured in this work, using the RVSAO package in IRAF.

${ }^{\mathrm{c}}$ From Hickson et al. (1989).

${ }^{\mathrm{d}}$ As derived in this work, not from RC3; see Section 4.4.

three-point dither pattern (sub-pixel dithering). The implementation of MultiDrizzle (Fruchter et al. 2009) in the HST/ACS data pipeline provides combined, geometrically corrected, and cosmic-ray cleaned images. For the analysis of point sources, we used the standard $H S T$ pipeline products with a nominal pixel scale of $0{ }^{\prime \prime} 05$ per pixel. For analysis of the extended sources, we 


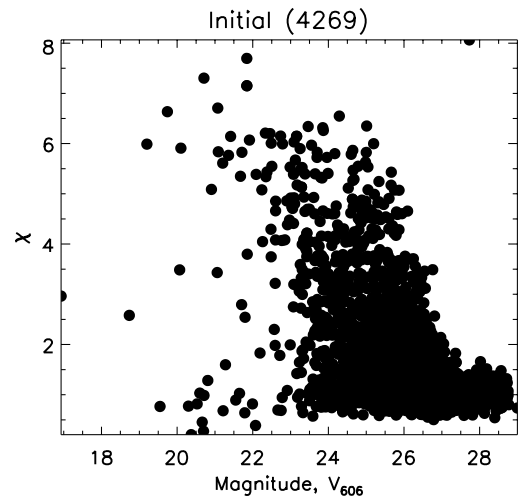

Magnitude, Sharpness \& $\mathrm{Chi}_{814}<3$ (3566)

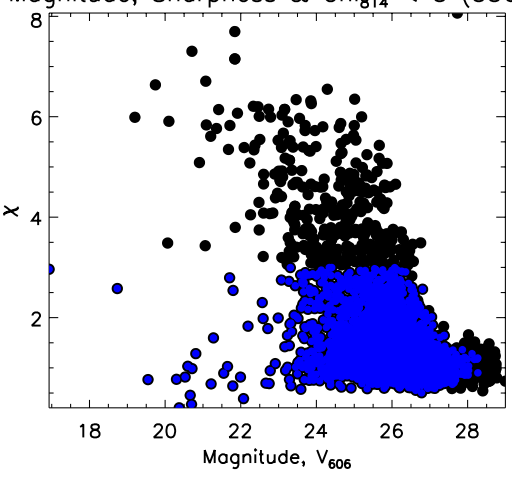

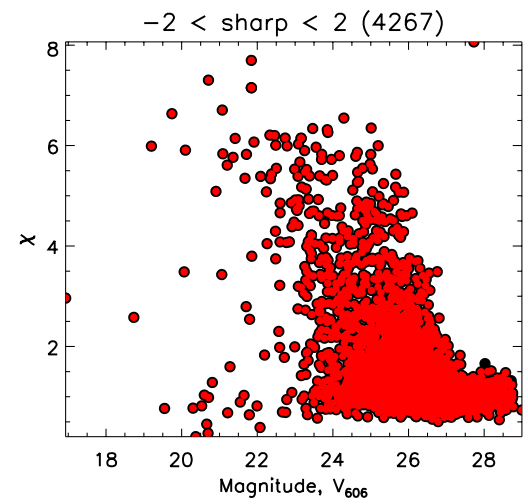

Magnitude, Sharpness, $\mathrm{Chi}_{814} \&$ color cut (3516)

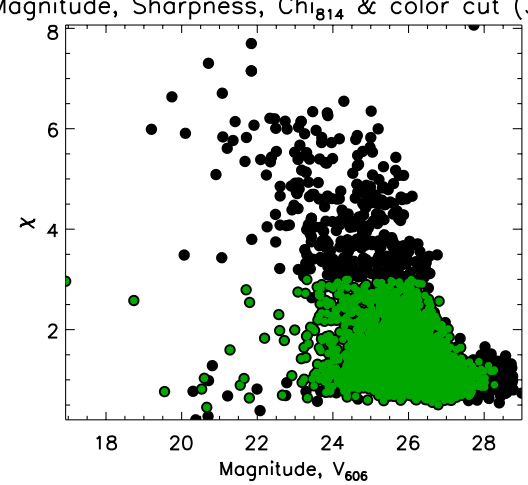

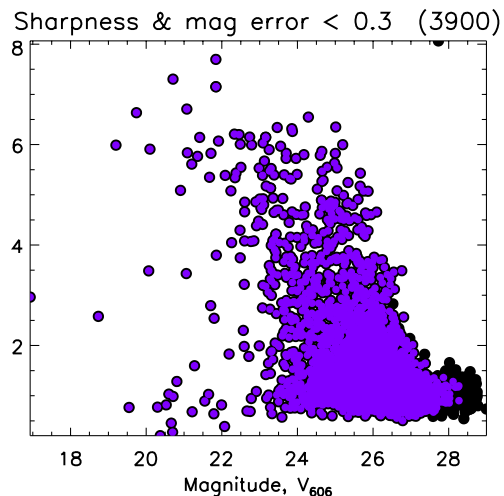

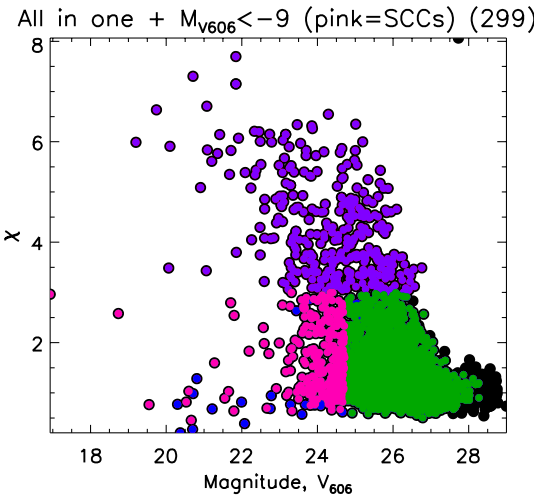

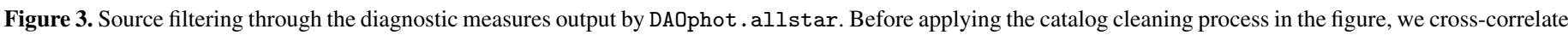

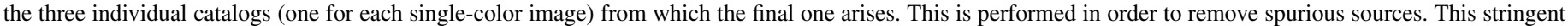
set of filters yields a somewhat low number of sources, which, however, make up a sample of bona fide clusters.

(A color version of this figure is available in the online journal.)

ran MultiDrizzle with the pixel scale set to 0. '03 per pixel to improve the spatial resolution.

The images, presented in Figure 2 (top panel), were used in a number of ways: to define the optical extent of galaxy disks and probe star formation in the IGM, to detect stellar aggregates and sub-galactic structures (i.e., individual star clusters and cluster complexes), and to obtain the magnitudes and colors of all these objects, and therefore estimate their ages. This enables the distinction between young and old clusters, i.e., the bright, blue young massive clusters (YMCs; also referred to as super star clusters, or SSCs) and fainter, red GCs.

\subsection{Selection and Photometry of Star Cluster Candidates}

At the adopted distance to HCG 7 of $56.6 \mathrm{Mpc}$ (Hickson et al. 1992), the only stars that are luminous enough to be detected are supergiants. Instead, star clusters are largely used as alternatives to individual stars in studying extragalactic star formation (e.g., Trancho et al. 2007a; Konstantopoulos et al. 2008, 2010; Konstantopoulos 2009; Bastian et al. 2009). Star clusters maintain a link to the overall star formation in any system (Lada \& Lada 2003) and can therefore be used to investigate the star formation history (e.g., Oestlin et al. 1998; Anders et al. 2004). Careful selection is essential, however, as clusters will appear as point sources on these images-at $56.6 \mathrm{Mpc}, 1$ pixel on the ACS chip corresponds to $\sim 11 \mathrm{pc}$, cf. the average star cluster half-light radius of $\sim 4 \mathrm{pc}$, as measured in a variety of environments, e.g., M101 (Barmby et al. 2006), M51 (Scheepmaker et al. 2007), and a selection of star-forming spirals (Larsen 2004).
We used the DAOphot package in $\operatorname{IRAF}^{17}$ to detect sources on the HST images, following the process presented in Gallagher et al. (2010): instead of applying a high detection threshold, we used a median-divided image to perform the initial selection. This prevents the loss of genuine clusters in high or varying backgrounds. We used a square of side 13 pixels to smooth the image and then divide the original by this smoothed image. Thus, the background is effectively lowered to unity (background divided by background) and the unresolved, marginally resolved, and extended sources can be distinguished. This also acts as a first filter against artifacts, as bright sources of limited extent will be effectively smeared out. After completing this transformation, we can use a low detection threshold to detect sources on the median-divided image.

A number of filters were applied to a detected source before it could be considered a star cluster candidate (SCC), which at this point did not differentiate between YMCs and GCs. At this first stage we used the $H S T$ weight maps to exclude spurious sources and we also restricted the selection to sources detected in all three filters.

We then proceed to apply a number of filters to eliminate sources of spurious or stellar nature, as well as background galaxies. We demonstrate the successive application of filters in Figure 3 and discuss the selection criteria below. Before applying the filters described in the following text, we crosscorrelate the $B_{435}, V_{606}$, and $I_{814}$ catalogs to remove spurious sources.

\footnotetext{
17 IRAF is distributed by the National Optical Astronomy Observatory, which is operated by the Association of Universities for Research in Astronomy, Inc., under cooperative agreement with the National Science Foundation.
} 


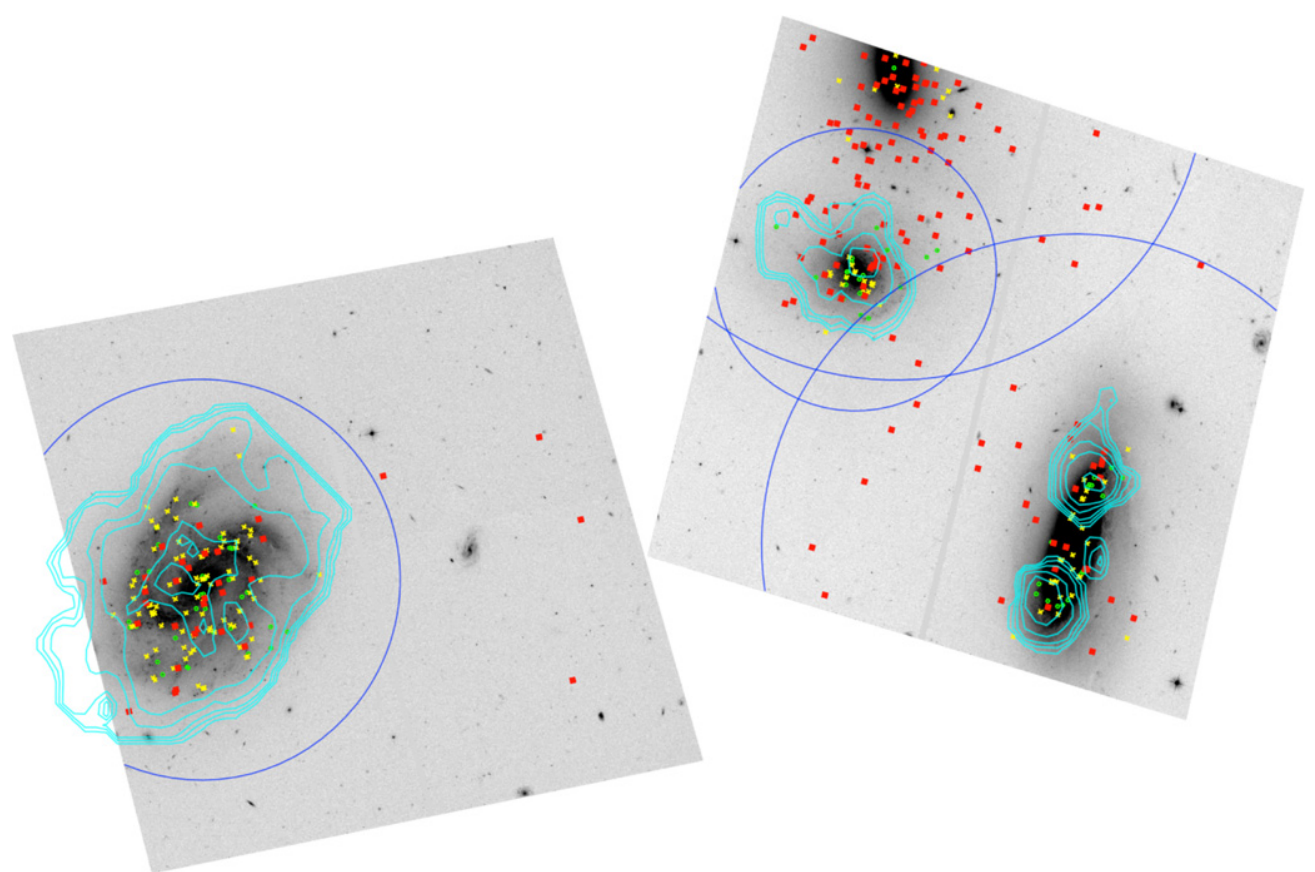

Figure 4. Spatial distribution of detected star cluster candidates across the group, color-coded according to age: open green circles and yellow crosses denote nebular and young SCCs, respectively, drawn from the final, $M_{V}<-9$ selected sample. The red boxes indicate globular cluster candidates, selected using a fainter brightness limit (see text for selection procedure). The large blue circles denote the limits of the galaxy halos, estimated according to galaxy mass. The proximity of $B$ and $D$ and consequent overlap of their halos makes it virtually impossible to distinguish between their respective GC systems. We have also plotted $\mathrm{HI}$ contours in cyan; the levels are $10,20,30,45,60,90$, and $120 \times 10^{19} \mathrm{~cm}^{-2}$. This shows the gas to be contained almost entirely within the individual galaxies (down to the sensitivity limit of the VLA observations), implying a lack of major interactions in recent times. The exception is $D$ that shows an asymmetric $\mathrm{H}$ I distribution. This is potentially the result of stripping from an encounter with $B$ sometime in the past $\sim$ Gyr (revisited in Figure 9 ).

(A color version of this figure is available in the online journal.)

With star clusters appearing as point sources, point-spread function (PSF) fitting needs to be conducted to obtain their photometric properties. A PSF was constructed for each filter using a number of bright, isolated stars with clear, smooth growth curves. We used daophot.allstar to conduct PSF photometry and applied aperture corrections to the photometry as the mean brightness difference of the stars we used to construct the PSF, between the 3 pixel PSF photometry and brightness measured in a 10 pixel aperture: $0.14,0.20$, and 0.21 in $B_{435}, V_{606}$, and $I_{814}$, respectively; the Sirianni et al. (2005) corrections were then applied to correct to infinity. Finally, foreground (galactic) extinction was accounted for using the standard Galactic extinction law (a correction of $A_{V} \sim$ 0.06 mag; Schlegel et al. 1998). We then restricted the SCC list further by imposing the following criteria: a "sharpness" (a measure of the relative width of a source with respect to that of the PSF) between -2 and 2, a photometric error below $0.3 \mathrm{mag}$ in all bands, and a $\chi$ goodness-of-fit statistic of less than 3 . This process follows the first steps of the selection routine tested by Rejkuba et al. (2005) for stars in resolved populations.

From this masterlist, samples of young and old cluster candidates can be drawn according to different luminosity and color cuts. To isolate young clusters, one needs to account for the contamination by individual giant stars. This is implemented through a (conservative) cut at an absolute magnitude of $M_{V}<$ -9 (standard practice, following the reasoning of Whitmore et al. 1999), in order to exclude the supergiants that populate the high end of the stellar luminosity function (red supergiants can reach $M_{V} \simeq-8$ mag; Efremov et al. 1986). The detection of GCs is simplified by their tight distribution in color space. Therefore, a lower magnitude limit can be enforced while still restricting stellar contamination.
The application of these stringent criteria produces a sample of 287 bright SCCs, of which almost half are situated in and around star-forming $C$. The locations of these sources are shown in Figure 4 and Figures 5 and 6 show the resulting color-color and color-magnitude diagrams for SCCs. Specifically, the numbers of detected SSCs in galaxies $A$ through $D$ are 50, 28, 133 , and 45 , with a further 31 objects in the space outside the galaxies.

\subsection{Completeness}

In order to test the completeness of our final list of SCCs, we used addstar to add 3000 artificial stars to the image (over the entire field, including superposed on the galaxies) in the magnitude range $24-28$, i.e., ( $-9.7,-5.7)$. The average limiting magnitudes for the $50 \%$ and $90 \%$ recovery rates are $(27.5$, $26.8),(27.5,26.3)$, and $(27.1,26.4)$ in the $B_{435}, V_{606}$, and $I_{814}$ bands, respectively (after photometric corrections are applied). Since this calculation represents an average over the entire field, we expect brightness completeness limits for clusters within galaxies. However, this is still far fainter than our $M_{V}<-9$ cutoff and does not affect the analysis.

\subsection{Globular Cluster Candidate Selection}

As contamination from luminous supergiants is not a problem for objects with GC-like colors, we adopt a fainter magnitude limit to select old GC candidates (GCCs) than we used for SCCs. We have chosen a cutoff at $V_{606}=26$, which corresponds to $M_{V} \sim-7.7$, or slightly more luminous than the expected peak in the GC luminosity function (GCLF) at $M_{V} \sim-7.4$ (e.g., Ashman \& Zepf 1998; Harris 2001). At this cutoff, the majority of GCCs lie above the $90 \%$ photometric completeness level in 

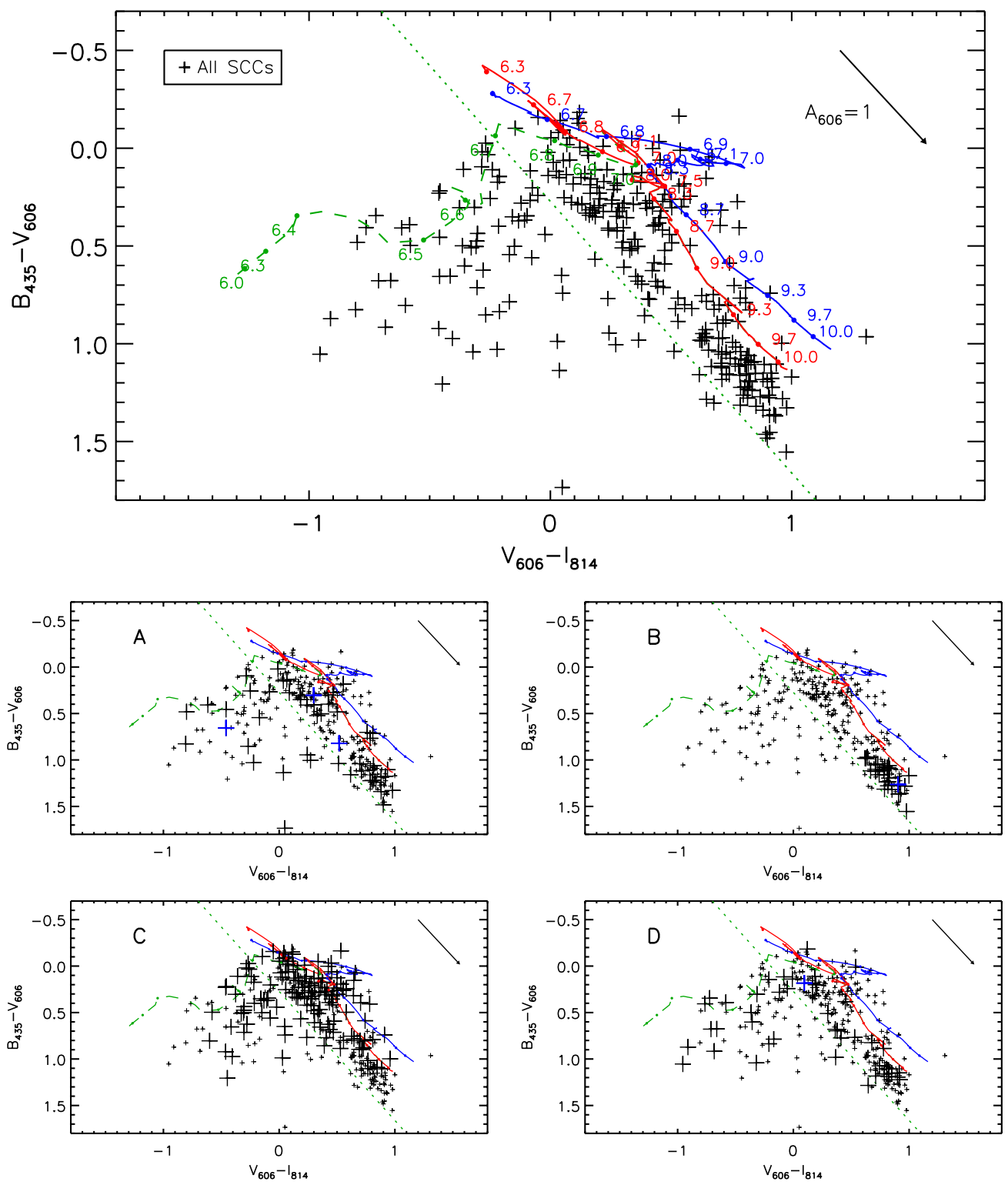

Figure 5. $B_{435}-V_{606}$ vs. $V_{606}-I_{814}$ colors of the entire sample of SCCs (top) and divided by regions (bottom), plotted on top of $\frac{1}{5} Z_{\odot}$ (solid red line, bottom) and $Z_{\odot}$ (solid blue) BC03 model tracks. The dashed green line shows an evolutionary track that accounts for nebular emission (SB99), as suited to the very youngest clusters that have not yet expelled their natal gas. All data points that lie to the left of the dotted green line are consistent with the nebular tracks, given an amount of extinction of no more than $\sim 1$ mag. SCCs (i.e., those with $M_{V}<-9$ ) are denoted by dark crosses; we indicate an extinction vector of length 1 mag in $V_{606}$. In the plots of individual galaxies, blue (bold) crosses mark the very brightest sources with $M_{V}<-11$, while we denote the entire sample as dots for comparison. A typical error bar is $\lesssim 0.1 \mathrm{mag}$ in either direction. All three spiral galaxies show signs of star formation over a Hubble time, as indicated by the spread of cluster colors about the model tracks. The lenticular $B$ only shows signs of cluster formation at ages greater than a few Gyr.

(A color version of this figure is available in the online journal.)

all three filters. Assuming a Gaussian GCLF with a peak at $M_{V}=-7.4 \pm 0.2$ and a dispersion $\sigma=1.2 \pm 0.2$, our cutoff allows us to sample $40 \% \pm 8 \%$ of the complete GCLF.

We have selected GCCs according to the color-space distribution of Milky Way GCs, as featured in Harris (1996). More specifically, we de-reddened the Harris catalog clusters by their listed $E(B-V)$ in the range $[0.00,1.24]$, and then defined a parallelogram based on the intrinsic $(B-V)$ versus $(V-I)$ color distribution of the MW GCs, and then transformed to the ACS filters using the "synthetic" transformations in Sirianni et al. (2005). All point sources with $1 \sigma$ error bars that overlap the color selection region are considered GCCs; they are shown in Figure 4 and their colors are plotted in Figure 7.
Much of the contamination in our GC sample will be either from foreground Milky Way stars or from reddened young clusters. Predictions from Milky Way star count models (the Besançon model of Robin et al. 2003) suggest that only 3-4 foreground stars will appear in the magnitude/color range for expected GCs in each of the ACS fields. Contamination from younger clusters is harder to discern, particularly in regions of the disks of the spiral galaxies where such objects should be common (Figure 4). To quantify, the youngest clusters with ages of $\tau \leqslant 10^{7} \mathrm{yr}$ (the ones most likely to suffer from high extinction) would need no more than an $A_{V} \lesssim 1.5$ mag to shift to the region occupied by GCs and fall within our criteria.

Due to the close (projected) proximity of the galaxies in the group (particularly $A / B / D$ ), it is likely that the halo GCs in 

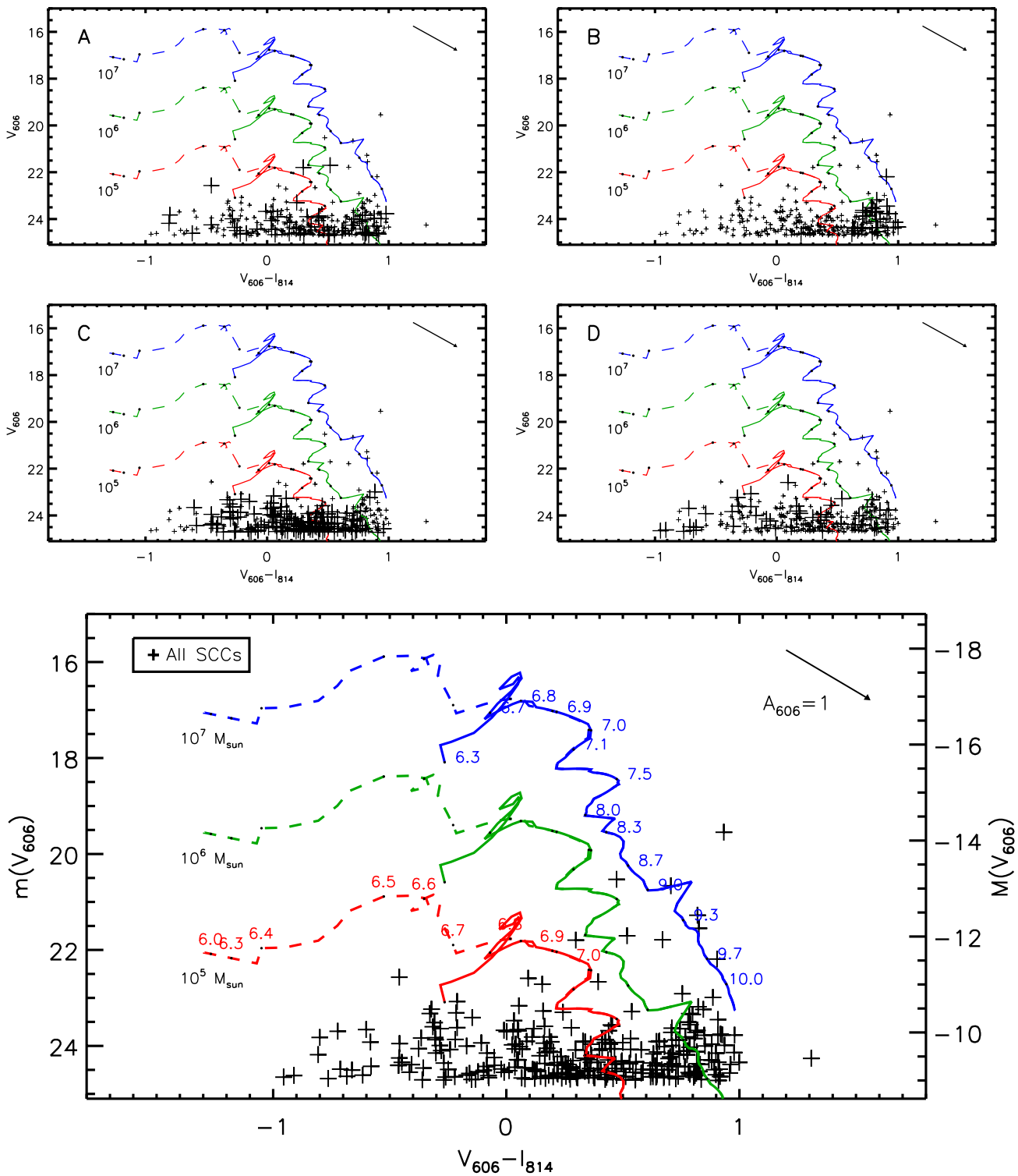

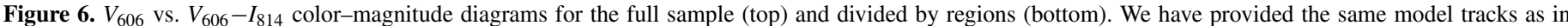

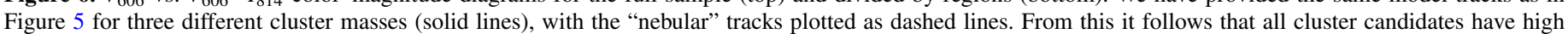

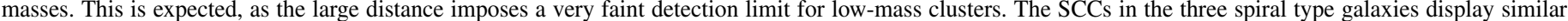
distributions in these plots, while in $B$ all SCCs are tightly concentrated. This is related to the narrow distribution of globular cluster colors.

(A color version of this figure is available in the online journal.)

each system will appear superposed. In an attempt to quantify the GCCs in each galaxy, we use the relationship between the galactic mass and the radial extent of the GC systems in galaxies by Rhode et al. (2007). To compute the expected size of each halo, we have adopted the mass-to-light ratio $(M / L)$ conversions assumed in that work, although we stress the general conclusions we reach are not dependent on the detailed size of any given halo. The expected sizes are $30 \mathrm{kpc}$ for $A$ and $B, 20 \mathrm{kpc}$ for $C$, and $14 \mathrm{kpc}$ for $D$; these radii are shown in Figure 4. While we cannot take into account any differences in the line-of-sight distances to each galaxy, the projected GC systems of $A, B$, and $D$ clearly overlap. We disentangle the systems in the subsections that follow.

The resulting GCCs are 21 for $A, 92$ for the overlapping systems of $B / D$, and 29 for $C$. These will be discussed in more detail in Section 5.

\section{ADDITIONAL OBSERVATIONS}

\subsection{Spitzer IRAC/MIPS Imaging in the IR}

The optical imaging was complemented by Spitzer imaging in the mid-IR (IRAC, $3.5-8 \mu \mathrm{m}$, MIPS $24 \mu \mathrm{m}$ ), presented in J07 and shown here in the bottom panel of Figure 2. The IRAC band covers stellar photospheric emission and probes the presence of polycyclic aromatic hydrocarbons (PAHs) and hot dust, while the $24 \mu \mathrm{m}$ observations trace thermal dust, all sources of emission stimulated by star formation activity.

The Spitzer images were combined with $J H K_{S}$ band observations from Two Micron All Sky Survey (2MASS; Skrutskie et al. 2006) to plot the IR spectral energy distribution (SED) of each galaxy (following J07), presented in Figure 8. We calculate the slope of the SED within the IRAC bands through a simple power-law fit. This was defined by Gallagher et al. (2008) as 

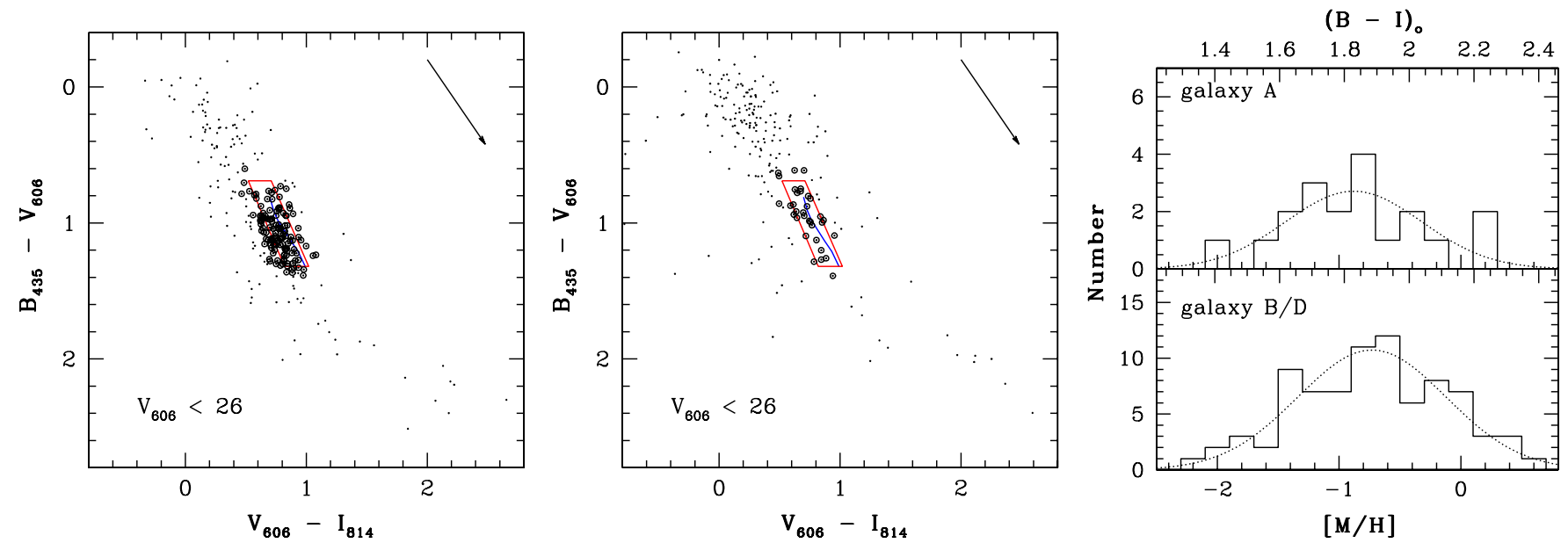

Figure 7. Left: color-color (CC) diagrams for globular cluster candidates in fields one and two, containing $A / B / D$ and $C$, respectively. We have indicated the precise boundaries of the GC selection box. The GC colors are consistent with Milky Way globulars. Right: the metallicity distribution of the $A$ and $B / D$ GC systems, as derived from the $B-I$ color. We find no evidence of bimodality, a trait attributed to mergers in the early history of galaxies (Muratov \& Gnedin 2010 ). This analysis could not be performed on the GC system of $C$, due to its face-on inclination (see Section 5).

(A color version of this figure is available in the online journal.)

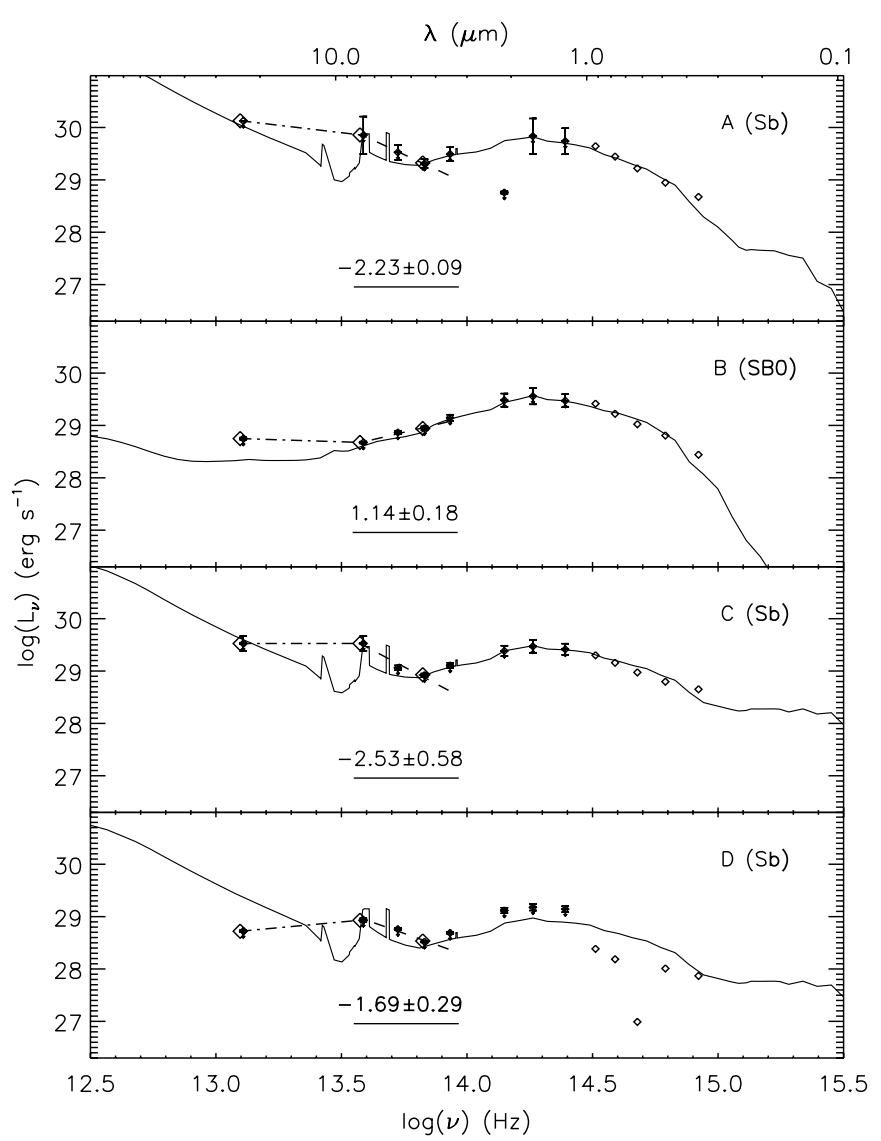

Figure 8. Optical-to-mid-IR SEDs for HCG 7 members. The photometric data, shown as diamonds, are drawn from the SDSS, 2MASS, and Spitzer MIPS/ IRAC. We annotate each panel with the adopted morphological type and with the value of the $\alpha_{\text {IRAC }}$ diagnostic (the line under the $\alpha_{\text {IRAC }}$ shows the fitting range). The lines represent GRASIL templates with parameters adapted to the derived characteristics of each galaxy (see Section 6). In all, the template fits reveal different spectral types than expected, based on morphology for $C, D$ and possibly also $A$. The lenticular $B$ is found to be best described by a young elliptical spectrum, $\tau=8 \mathrm{Gyr}$. The template plotted for $A$ can be replicated by maintaining the original Sa designation and enhancing the star formation by a factor of $\sim 5$. The SED of $D$ is difficult to model, perhaps due to its previous misclassification as SB0. the $\alpha_{\text {IRAC }}$, and it serves as a measure of star formation activity. The extent of the flux difference between the PAH features at $8 \mu \mathrm{m}$ and the stellar-blackbody emission at $3.5 \mu \mathrm{m}$ leads to a positive gradient (blue color) in quiescent environments, while star formation registers as a negative slope (red color). The steepness of the slope scales with the intensity of the star formation.

\subsection{VLA $21 \mathrm{~cm}$ Observations: Derivation of Gas Mass and Column Density}

We also make use of radio wavelength observations, in order to investigate and characterize the state of neutral hydrogen gas in the system. While H I observations of HCG 7 have been presented in the past (Huchtmeier 1997; Verdes-Montenegro et al. 2001; Borthakur et al. 2010), these single-dish data covered the system as a whole and not the member galaxies on an individual basis. We used archival Very Large Array (VLA) H I data, obtained in the $C$ and $D$ array configurations, to map the column density of $\mathrm{H}_{\mathrm{I}}$ across the system and to derive the masses of all member galaxies.

The reduction and analysis of these data will be presented in full in A. Heiderman et al. (2011, in preparation, hereafter H11); we will refer to this work in the following sections. In brief, we used standard techniques and procedures in the Astronomical Image Processing System (see Rupen 1999) to reduce the data. The data contained major interference issues, which were carefully corrected before calibration and bandpass correction. The data were continuum subtracted by making a linear fit to the visibilities, and then combined in the UV plane. The $C+D$ combined data have a spatial resolution and channel separation of $19^{\prime \prime} .4 \times 15^{\prime \prime} .2$ and $21.3 \mathrm{~km} \mathrm{~s}^{-1}$. The on-source integration time totaled $\sim 7 \mathrm{hr}$ and the number of combined spectral channels were 54 . This corresponds to a $1 \sigma$ rms noise of $0.3 \mathrm{mJy} \mathrm{beam}^{-1}$ and a $\sigma\left(N_{\mathrm{HI}}\right)$ column density sensitivity of $2.4 \times 10^{19} \mathrm{~cm}^{-2}$ per channel over the beam size.

We used a moment zero map to derive a mapping of column densities and an estimate of the $\mathrm{H}_{\mathrm{I}}$ mass contained in each galaxy. We follow a simple transformation of radio intensity $\left(S_{v}\right)$ to brightness temperature $\left(T_{b}\right)$ and then to neutral hydrogen 

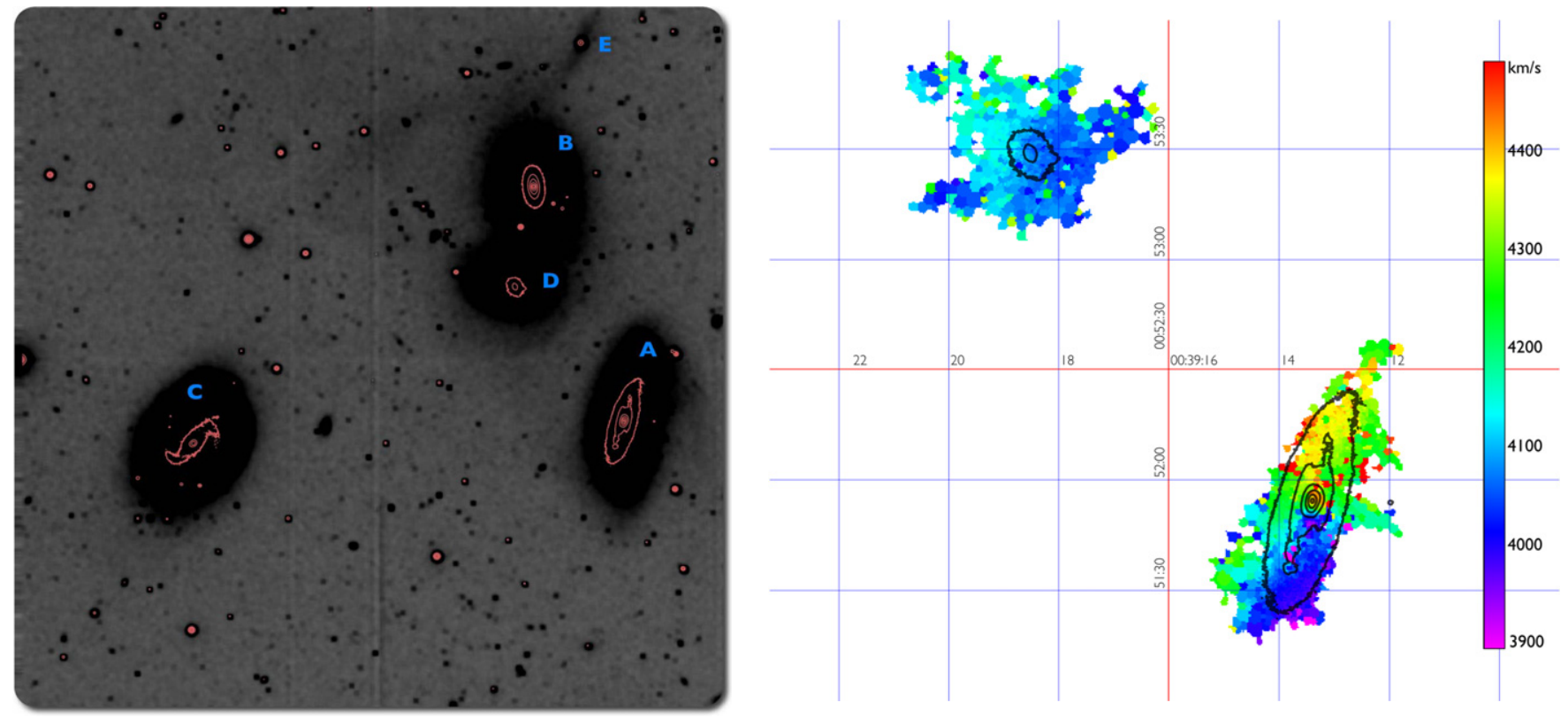

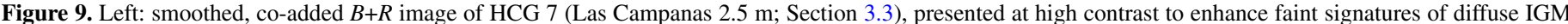

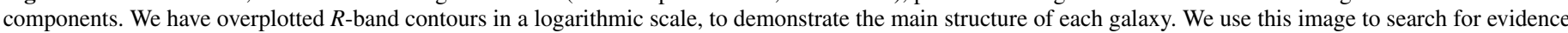

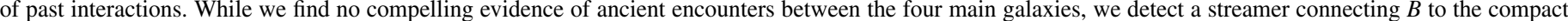

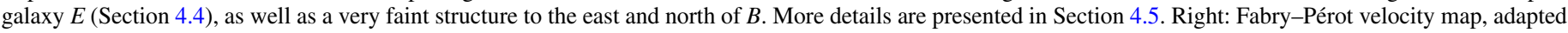

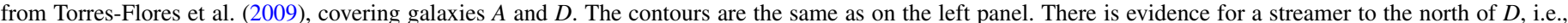

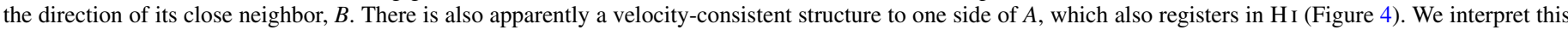
collimated stream as a galactic wind emanating from the nucleus of $A$ (Section 6).

(A color version of this figure is available in the online journal.)

column density $\left(N_{\mathrm{H}_{\mathrm{I}}}\right)$ and mass $\left(M_{\mathrm{H}_{\mathrm{I}}}\right)$ :

$$
\begin{aligned}
T_{b} & =S_{v} \times c^{2} /\left(2 v^{2} k\right) \mathrm{K} \\
N_{\mathrm{HI}} & =1.8224 \times 10^{18} \times \int T_{b} d v \mathrm{~cm}^{-2} \\
M_{\mathrm{HI}} & =2.36 \times 10^{5} \times d^{2} \int S_{v} d v M_{\odot},
\end{aligned}
$$

where $c$ is the speed of light in vacuum and $k$ is the Boltzmann constant.

The derived column densities are shown as green contours in Figure 4 and masses of galaxies $A, C$, and $D$ were calculated as $\log M_{\mathrm{HI}}=9.0,9.5,8.9 M_{\odot}$; no $\mathrm{HI}$ gas was detected in the vicinity of $B$.

\subsection{Las Campanas Wide-field Imaging}

The imaging coverage is extended through our wide-field mosaic imaging of the group and its surroundings in the $B$ and $R$ bands. These images were obtained with the Direct CCD Imager on the Du Pont $2.5 \mathrm{~m}$ telescope at Las Campanas, Chile on the nights of 2007 October 3 and 5 under good seeing conditions (approximately 0.'75), as part of a project covering all 12 HCGs in our sample. The exposure times were 180 and $120 \mathrm{~s}$ with the $B$ (JB 3008) and $R$ (KC-R 3010) filters, respectively, allowing for point sources to be detected down to roughly $24 \mathrm{mag}$ (in either filter) at $\mathrm{S} / \mathrm{N}=5$.

These images are used to look for signatures of ancient interactions, a subject we discuss in full in Section 4.5 (and Figure 9). Their primary purpose was, however, to provide a list of candidate dwarf galaxies in HCG 7 for spectroscopic followup. This program is ongoing with the Hydra spectrograph on the Cerro Tololo Inter-American Observatory (CTIO) $4 \mathrm{~m}$ telescope and will be presented in full once concluded. A brief account of the data acquisition and reduction is offered in the following section.

\subsection{CTIO-Hydra Multi-Object Optical Spectroscopy}

The data set is completed by optical spectroscopy of candidate dwarf galaxies selected through the Las Campanas imaging campaign presented in Section 3.3. This ongoing spectroscopy project will eventually cover all compact groups in our multiwavelength data set and will be presented in full in a later work. The HCG 7 data set was obtained with the Hydra multi-fiber spectrograph on the CTIO $4 \mathrm{~m}$ telescope. Observations were carried out between 2008 August 3 and 10 with a total on-target integration time of $\sim 13.5 \mathrm{hr}$, with various fiber configurations, covering a different set of targets in each exposure. We used grating KPGL2 with the GG 385 blocking filter (position 2) to avoid order contamination. The SITe CCD was binned by a factor of 2 in the dispersion direction to reduce readout noise. The resulting spectra cover the range $\sim 3000-7800 \AA$ at a dispersion of $2.3 \AA$ pixel $^{-1}$. The resolution, measured as the full width at half maximum of a skyline at $\sim 5000 \AA$, is $6.1 \AA$. Wavelength calibration was facilitated through exposures with the $\mathrm{He}-\mathrm{Ne}-\mathrm{Ar}$ penray lamps. We took twilight flats to compensate for fiber-to-fiber throughput variations and "milky flats" to remove the two-dimensional instrumental spectral shape. ${ }^{18}$ We used the Hydra-specific software hydraassign to construct fiber configurations for targets, guide stars, and blank sky observations. Guide stars were selected from the Sloan Digital Sky Survey (SDSS; York et al. 2000) that fully covers the HCG 7 field. Each configuration included at least

\footnotetext{
18 This is a daylight flat that is taken in order to illuminate wavelengths that register very faintly under the artificial light of calibration lamps. As the daytime sky is warmer than a lamp, we use that to obtain a smooth flat-field division across the broad wavelength range covered.
} 

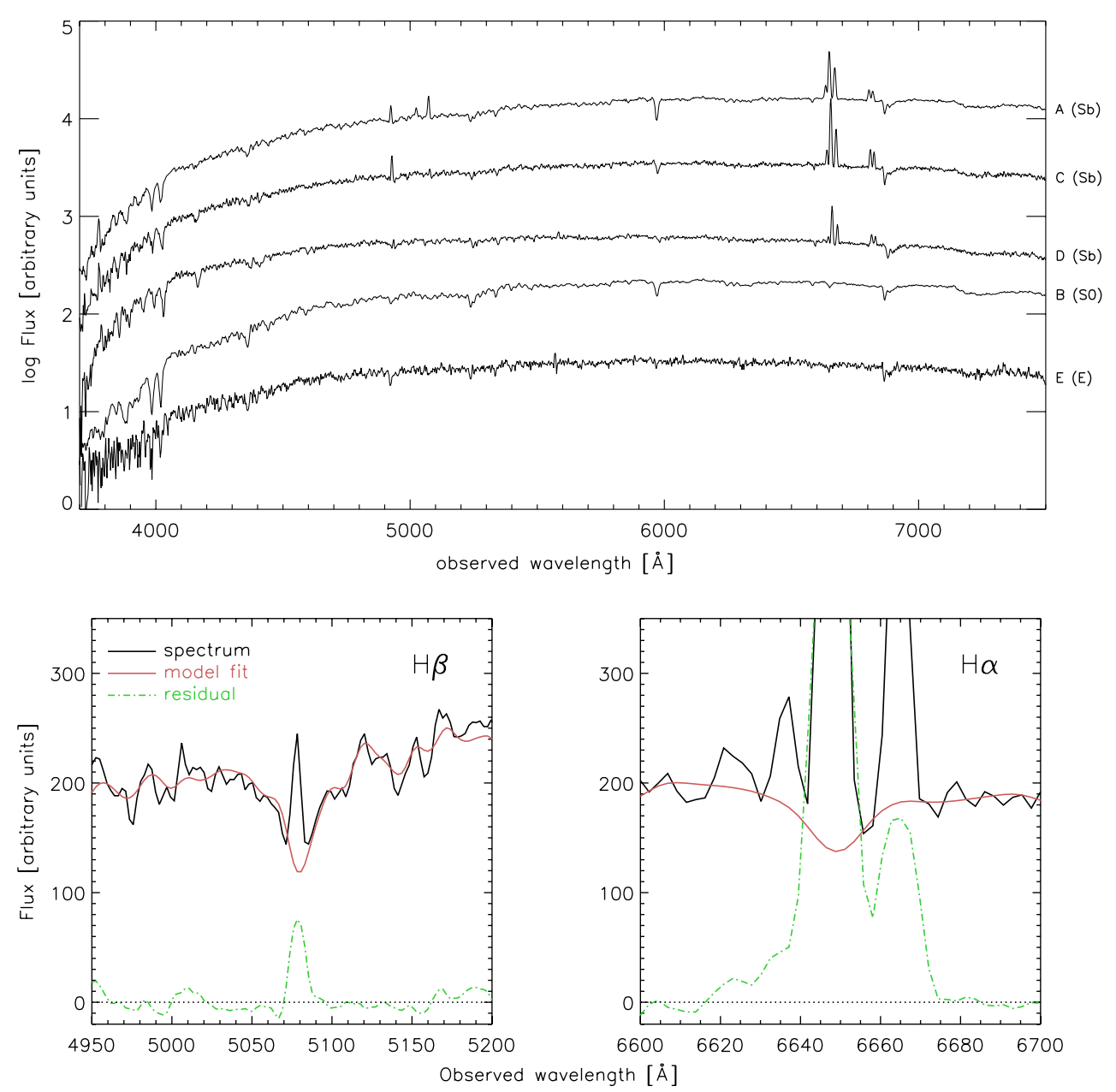

Figure 10. Top: CTIO-Hydra optical spectra of the newly discovered compact galaxy $E$ - the only one of its kind detected down to our $R \sim 20.5$ spectroscopic limit - and the nuclei of the four main HCG 7 galaxies. The flux axis is plotted in logarithmic space to ease presentation and comparison. The spectra of spiral galaxies $A / C / D$ all exhibit $\mathrm{H} \alpha$ emission. The $[\mathrm{O} \mathrm{III}] / \mathrm{H} \beta,[\mathrm{N} \mathrm{II}] / \mathrm{H} \alpha$, and $[\mathrm{S} \mathrm{II}] / \mathrm{H} \alpha$ emission line ratios of $A$ place it in the $\mathrm{H}$ II regime (see Section 6), indicating a high ionization continuum in its core. $C$ shows a spectrum more appropriate for an Sb galaxy, while $D$ exhibits absorption in $\mathrm{H} \beta$, with a weak, embedded emission component, uncharacteristic of an Sc; we discuss the nature of this galaxy as a spiral at the verge of becoming a lenticular in Section 6. The nucleus of $B$ shows an elliptical-type spectrum, with deep absorption lines and no emission, as does the compact galaxy $E$, albeit at a significantly lower $\mathrm{S} / \mathrm{N} \sim 20$. The classification of all galaxies is discussed in Section 6 and Figure 8. In the bottom panels, we demonstrate the "E+A" spectrum of $D$ : the separate lines show the spectrum and pPxF model fits (see Section 6.4) of the $\mathrm{H} \beta$ and $\mathrm{H} \alpha$ regions.

(A color version of this figure is available in the online journal.)

12 guide stars and 30 blank sky observations and a list of targets tiered according to magnitude, in order to optimize exposure times. We thus produced a set of five configurations that included progressively fainter targets, with the aim of obtaining a complete, flux-limited set of observations for our targets. We estimate that our observations for HCG 7 targets are complete down to $R \sim 20.5$.

Data reduction was carried out using the standard IRAF ccdred package as well as the dedicated IRAF package hydra. We used LACOSMIC (van Dokkum 2001) to eliminate cosmic rays in individual exposures before spectral extraction. After spectral extraction, we used custom-written IRAF scripts to combine all data and error spectra at the same configuration, weighting them by their variance.

In HCG 7, we identify a single additional member with high confidence, a compact galaxy to the northwest of $B$, at a projected distance of $\sim 30 \mathrm{kpc}$. This one source results from a list of hundreds of candidates down to $R \sim 20.5$. Its spectrum is shown in Figure 10.

We also used this program to place fibers on the four main galaxies, also shown in Figure 10 . The $2^{\prime \prime}$ width of the Hydra fiber translates to $\sim 0.8 \mathrm{kpc}$ at the distance of HCG 7, therefore the spectra cover the central regions of the galaxies. A quick inspection shows features inconsistent with their previous designation of morphological types: $A$ shows very bright emission in $\mathrm{H} \alpha$ and some in $\mathrm{H} \beta$, features not typical of an $\mathrm{Sa}$; the spectrum of $C$ is not dominated by the blue continuum, as one would expect from an $\mathrm{Sc}$; while $D$ features $\mathrm{H} \alpha$ emission, it is embedded in a deep stellar absorption line, indicative of a post-burst spectrum. The spectroscopic classification of HCG 7 galaxies will be revisited in Section 6, where we present a combined analysis of their spectra and optical-to-mid-infrared SEDs.

\section{THE PRESENT STATE OF HCG 7: GAS CONTENT AND STAR FORMATION ACTIVITY}

The current consensus in star formation research suggests that the vast majority of star formation occurs in a clustered fashion. Owing to this fact, the processes of star and cluster formation are inextricably linked. The cluster population of a galaxy therefore provides information on its current state and recent star formation history. Here, we will provide an interpretation 
of the photometric properties of the detected SCCs in HCG 7. We combine this information with overall galaxy properties to draw implications on the evolution of this compact group.

\subsection{Star Clusters}

We compared the colors of all SCCs to models of simple stellar populations (SSPs) by Bruzual \& Charlot (2003). These models only predict properties of the stars, and do not incorporate either continuum or line emission from the gas that surrounds very young clusters. We assume a metallicity of $\frac{1}{5} Z_{\odot}$ for all four galaxies, following the mass-metallicity relation observed in star-forming galaxies (Ellison et al. 2009). Unfortunately, as our analysis lacks $U$-band imaging, we are unable to break the age-extinction degeneracy characteristic for SSPs with ages $\tau \lesssim 1$ Gyr. The model track runs virtually parallel to the extinction vector, hindering the distinction of old clusters from reddened younger clusters based on colors alone. However, the goals of this study can be met through a qualitative age-dating process: we are investigating distinct epochs of star formation and those can still be identified.

This analysis is presented in Figures 5 and 6, where we show the $B-V$ versus $V-I$ color plots and corresponding color-magnitude diagrams. The large plots show the collective properties of the HCG 7 cluster population, while the smaller panels pertain to individual galaxies. Each of these panels shows the "footprints" of all SCCs in HCG 7 as dots, with individual galaxy members denoted by crosses. Blue (thick) crosses indicate the very brightest sources, those with $M_{V}<-11$.

Crucially, the very youngest clusters can be distinguished by their strong nebular emission, caused by the ionization of their surrounding gas. This is either the residual gas of star formation that is yet to be expelled, or gas in the general vicinity of a cluster-forming region, both indicative of a young age (although line-of-sight effects are possible in the latter case). The result of this is emission in $\mathrm{H} \alpha$ and [O III], both covered in the $F 606 W$ filter $\left(z_{\text {HCG } 7} \simeq 0.014\right.$, i.e., a wavelength-space shift of $\Delta \lambda \simeq 90 \AA$ ).

In order to incorporate that information, we obtain the strength of the $\mathrm{H} \alpha$ and $\mathrm{H} \beta$ emission lines from Starburst99 models (Leitherer et al. 1999, SB99) with the same metallicity and initial mass function. As SB99 does not include the [O III] line, we defined it through the ratio of $\log ([\mathrm{O} \mathrm{III}] / \mathrm{H} \beta)$. We used the highest value for this diagnostic of 0.7 (as found in the KISS sample of low-mass star-forming galaxies; Salzer et al. 2005) to estimate the temporal evolution of the [O III] emission line strength. This is, of course, a qualitative treatment of the problem at hand: the relative strength of $[\mathrm{O} \mathrm{III}] / \mathrm{H} \beta$ is environment and metallicity dependent and mildly varies from one cluster to the next, according to the properties of the gas (Östlin et al. 2003). There are more lines that should be considered for a robust treatment of this issue, such as [O II].

In effect, our treatment is sufficient to separate "nebular" sources: young clusters with nebular emission can be distinguished from older clusters by roughly dividing color space into regions. First, we draw a line parallel to the extinction vector, crossing the nebular model track at $\log \tau=6.7$ (the dotted green line in Figure 5). We consider any source to the left of this line to have nebular emission, i.e., to belong to a clusterforming region, and therefore assign an age of $\lesssim 10$ Myr. We must caution at this point that $\mathrm{H} \alpha$ emission does not prove that a source is younger than $10 \mathrm{Myr}$, but solely that it is surrounded by ionized gas. Such chance alignments have been found to be a common occurrence in nearby environments by Trancho et al. (2007b) and Konstantopoulos et al. (2010), as deduced through the spectroscopic examination of the absorption (stellar) and emission (nebular) line signatures. This might arise from the limited spatial resolution of the ground-based observations of the aforementioned works. The issue is treated in Whitmore et al. (2010), where it is found to have a limited impact in high-resolution, $H S T$-based studies. In addition, the absence of high-resolution $U$-band imaging disqualifies precise cluster age dating on an individual basis. A cluster that is found in an $\mathrm{H} \alpha$ bright region is most likely to be no more than a few tens of Myr old, an age space that cannot be resolved by our diagnostics.

Another region is drawn to distinguish GCCs. This rectangle is based on the observed colors of Milky Way GCs (Harris 1996) and is indicated on Figure 7. Any source not belonging to one of these regions is considered a candidate YMC. Note that we also apply a color cut at $B_{435}-V_{606}<1.5 \mathrm{mag}$ or $V_{606}-I_{814}$ $<1 \mathrm{mag}$ to exclude foreground stars. This process was outlined in Section 2.3.

The vast majority of young clusters (nebular included) are located in or around the actively star-forming spirals. In Figure 4, we show the spatial distribution of all cluster candidates, coded in green, blue and red for nebular, young and old, respectively. As expected, the lenticular $B$ is found to host the largest population of old clusters, In fact, $B$ hosts no detectable candidates aged less than a few $\mathrm{Gyr}$, implying that star formation slowed down significantly long ago.

The color-color plots of the spiral galaxies reveal an apparent lack of clusters at $\tau \sim 1$ Gyr. This is a common feature in populations of star clusters and arises primarily because of the evolutionary fading of stellar aggregates: with stellar evolution gradually extinguishing the most luminous stars, the $M / L$ of SSPs increases steadily with time. If $M / L \propto \tau^{\alpha}$, then $L \propto M / \tau^{\alpha}$, meaning that a constant magnitude cutoff will restrict our sample to higher mass with increasing cluster age. This results in fewer clusters being selected once we cross the Gyr mark. In addition, the lack of $U$-band observations inhibits the distinction between an old cluster and a reddened younger cluster. It takes less than 2 mag of extinction to displace a cluster from $\log \tau \sim 7$ to $\log \tau \sim 10$. In that way, a young cluster in a dusty star-forming region may mimic a globular.

\subsection{Cluster Complexes}

Star clusters generally do not form in isolation. Like the stars they contain, clusters represent a level of hierarchical structure formation within galaxies. It is believed that the majority of clusters form in complexes that cover a large range of masses and sizes. This connection was explored by Elmegreen \& Elmegreen (1983), who found that spiral arms provide the appropriate conditions for the largest complexes to form. As these gas agglomerates can reach masses of $\sim 10^{7} M_{\odot}$, the high end of their mass distribution can be observed to great distances. In nearby environments, the size and mass distributions are better sampled, and relations between their physical properties can be pursued to greater detail. Bastian et al. (2005) found them to obey a mass-radius relation of the form $M \propto R^{2.33 \pm 0.19}$, a link that is communicated between complexes and GMCs $\left(M_{\mathrm{GMC}} \propto R_{\mathrm{GMC}}^{2}\right.$; Solomon et al. 1987).

As complexes represent such a fundamental part of the star formation process, they merit an in-depth study. At large distances, however, their detection is non-trivial: the spatial resolution limit of even $H S T$ gives rise to sample contamination, especially in inclined spirals, where chance alignments are common. 

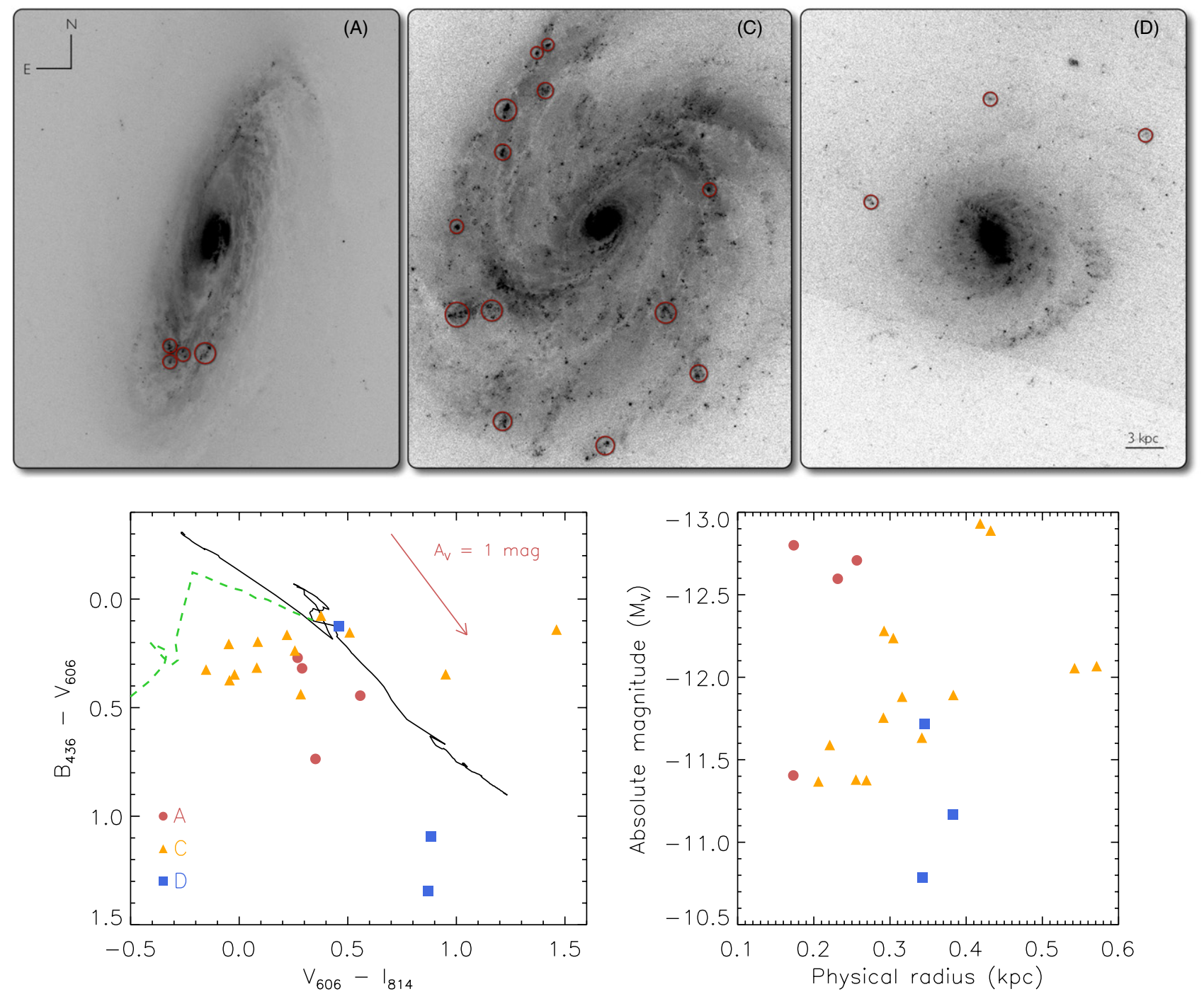

Figure 11. Star cluster complexes in HCG 7 (HST $V_{606}$ images). In the top panel, we have indicated their locations in each of the three star-forming galaxies (presented to scale). These structures represent the highest level of the star formation hierarchy within galaxies. They contain a number of star clusters and represent more than a single epoch of cluster formation. In accord with this view, we find the complexes to have colors, and therefore ages, consistent with young stellar populations-as presented in the bottom left panel. Their absolute magnitudes are plotted against their sizes in the bottom right panel; the magnitudes scale approximately with galaxy brightness, $V_{606}=13.4(A), 13.8(C)$, and $15.4(D)$. Complexes in $A$ are difficult to measure because of inclination effects.

(A color version of this figure is available in the online journal.)

In this work, we studied of the properties of 21 complexes- 4 , 14 , and 3 in $A, C$, and $D$, respectively, shown in Figure 11. We selected them by eye (the large pixel scale at this distance impedes automatic detection), roughly measured their sizes as the extent that lies about $10 \sigma$ above the background, and compared their colors (after background subtraction) to SSP models in order to obtain age estimates. While the possibility of contamination is quite high in the inclined $A$, the sizes and colors of the detected groupings are consistent with the distributions established in the local universe (Elmegreen \& Salzer 1999). More specifically, most complexes have colors indicating nebular emission features, as expected by their high gas content and young ages; that is to say, they stray to the left of the model track, as do gas-enshrouded clusters (Figure 11). Two of the three complexes in the inclined spiral $A$ appear to be heavily reddened $\left(A_{V} \sim 2.0 \mathrm{mag}\right)$, in tune with their locations in the galaxy.

In all, the sizes and colors of complexes in the three galaxies are consistent with quiescent systems found locally (see above references). This is to be expected, as the size of complexes is only dependent on that of the parent galaxy. The fact that we detect young star clusters of various ages associated with these complexes supports their role as the primary sites of star cluster formation (ongoing until depletion) and therefore the hierarchical nature of star formation in galaxies.

\subsection{Star Formation Rates and Stellar Masses}

Key to deciphering the present state of the system are the SFRs of the individual galaxies. For this, we adopt the measurements of Tzanavaris et al. (2010, hereafter T10). That work combines the Spitzer $24 \mu \mathrm{m}$ described in Section 3.1 with $2000 \AA$ photometry from the Swift ultraviolet and optical telescope (UVOT; Roming et al. 2005) to derive the "true" SFR. The two wavebands capture different stages of star formation: the IR probes star-forming clouds, where protoclusters can be found deeply embedded in their natal clouds (Lada \& Lada 2003). The high column densities of dust in these clouds absorb 
Table 2

Derived Photometric and Other Information

\begin{tabular}{|c|c|c|c|c|c|c|c|c|c|c|}
\hline ID & $\begin{array}{c}\text { Dimensions } \\
(\mathrm{kpc})\end{array}$ & $\begin{array}{c}\log M_{*} \\
\left(M_{\odot}\right)\end{array}$ & $\begin{array}{c}\log M_{\mathrm{H}_{\mathrm{I}}} \\
\left(M_{\odot}\right)\end{array}$ & $\begin{array}{c}\mathrm{SFR} \\
\left(M_{\odot} \quad \mathrm{yr}^{-1}\right)\end{array}$ & $\begin{array}{c}\text { SSFR } \\
\left(\times 10^{-11} \mathrm{yr}^{-1}\right)\end{array}$ & $\begin{array}{c}\log \Sigma_{\mathrm{SFR}}{ }^{\mathrm{a}} \\
\left(M_{\odot} \mathrm{yr}^{-1} \mathrm{kpc}^{-2}\right)\end{array}$ & $\begin{array}{c}\log \Sigma_{\mathrm{H}_{\mathrm{I}}}{ }^{\mathrm{a}} \\
\left(M_{\odot} \mathrm{pc}^{-2}\right)\end{array}$ & $N_{\text {SCC }}$ & & \\
\hline A & $31 \times 15$ & 10.22 & 9.0 & $3.88 \pm 0.47$ & $23.46 \pm 4.25$ & -2.3 & 0.1 & 50 & 0.4 & 0.2 \\
\hline B & $20 \times 13$ & 9.95 & $<8.1^{\mathrm{b}}$ & $0.23 \pm 0.02$ & $2.56 \pm 0.42$ & -3.1 & -0.4 & 28 & 2.5 & $0.9^{c}$ \\
\hline $\mathrm{C}$ & $29 \times 19$ & 9.88 & 9.5 & $2.06 \pm 0.17$ & $27.33 \pm 4.34$ & -2.5 & -2.7 & 133 & 0.3 & 0.2 \\
\hline $\mathrm{D}$ & $16 \times 13$ & 9.56 & 8.9 & $0.43 \pm 0.04$ & $11.89 \pm 1.90$ & 0.7 & 0.6 & 45 & 2.5 & $0.9^{c}$ \\
\hline$\Sigma$ & $200 \times 120$ & 10.56 & 9.7 & $6.60 \pm 0.50$ & \pm & & & 256 & & \\
\hline
\end{tabular}

Notes.

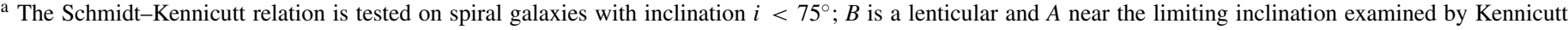
(1998).

b There is no detection of $\mathrm{H}_{\mathrm{I}}$ in lenticular $B$ in our VLA data; this value represents an upper limit, based on the sensitivity of our observations.

c The GC systems of $B$ and $D$ are overlapping; this is the combined number.

the UV photons emanating from protostars, become thermally excited, and re-emit the radiation at longer wavelengths. The timescale for this phase (the age of a protocluster) is $\tau_{\mathrm{IR}} \lesssim$ 1 Myr, thus the mid-IR SFR measures the current SF activity. At a timescale of $\sim 100 \mathrm{Myr}$, the clouds have been expelled by the mechanical feedback of stellar winds and supernovæ and the UV photons, so richly emitted from the photospheres of young stars, are free to escape along many lines of sight. The SFR derived from the UV is therefore telling of a more prolonged era of star formation and is appropriate for studying quiescent environments. It does, however, miss information of current activity ( $\tau \lesssim 10 \mathrm{Myr}$ ) that is dust enshrouded and therefore emerges at longer wavelengths.

T10 calculate the rates of $A / B / C / D$ as $3.88 \pm 0.47,0.23 \pm$ $0.02,2.06 \pm 0.17$, and $0.43 \pm 0.04 M_{\odot} \mathrm{yr}^{-1}$. Given the variation in galaxy sizes, we can relate the SFRs to the stellar mass to get a better handle on the current star formation activity in the HCG 7 galaxies. T10 divide by the stellar mass of each galaxy, as derived from 2MASS $K_{S}$-band photometry (see J07, Table 2), to obtain specific star formation rates (sSFR) of $23.46 \pm 4.25$, $2.56 \pm 0.42,27.33 \pm 4.34$, and $11.89 \pm 1.90 \times 10^{-11} \mathrm{yr}^{-1}$. The relation between disk-averaged SFRs and $M_{\mathrm{HI}}$ is in all cases consistent with the correlation found in Kennicutt (1998).

Comparing to the SINGS data set (Kennicutt et al. 2003), these rates suggest the three disk galaxies are moderately star forming, with SFRs at the high end of the quiescent regime, given their morphologies, sizes, and stellar content. The lenticular $B$ is also in tune with its morphological counterparts, again, at the high end of the SFR distribution. Comparing to the sample of nearby galaxies treated by Leroy et al. (2008), the sSFRs are comparable to those of star-forming galaxies in that sample-although because of the low numbers, this is not a statistically significant comparison. This indicates relatively high star formation activity throughout the group. Finally, we compare to the AMIGA sample of isolated galaxies (Verdes-Montenegro et al. 2005; Lisenfeld et al. 2007), which, unfortunately, does not have associated stellar mass information. A comparison based on morphological type alone places the SFRs $1 \sigma$ above the median values of 0.72 and $1.09 M_{\odot} \mathrm{yr}^{-1}$ for Sa and Sc types, respectively, where the corresponding standard deviations are 1.22 and $2.81 M_{\odot} \mathrm{yr}^{-1}$.

All the values quoted in this section are listed in Table 2.

\subsection{Detection of Compact Galaxies}

Our spectroscopic campaign to identify group members further from the four main galaxies (Section 3.4) yielded a single high-confidence detection. This is a compact galaxy to the northwest of $B$, apparently associated with a stellar streamer (Section 4.5) to which we refer as $E$. It was detected in the SDSS, where it cataloged as J003915.46+005633.2. Its spectrum is shown in Figure 10 and its radial velocity is measured as 4118 $\mathrm{km} \mathrm{s}^{-1}$ (determined through the $\mathrm{C}$ II $\mathrm{H}$ and $\mathrm{K}$ lines). The spectrum is characteristic of an old elliptical galaxy, with no discernible emission features, as also confirmed by the SDSS spectrum. Its redshift gives rise to a luminosity distance of $D_{L}=57.8 \mathrm{Mpc}$ and therefore an absolute blue magnitude of $M_{B}=-15.2 \mathrm{mag}$.

Its brightness and spectrum are consistent with a dwarf elliptical galaxy. Its spectrum will be revisited in a later work, where we will be examining the dwarf galaxy populations of all HCGs in our multi-wavelength sample.

\subsection{Searching for Optical Signatures of Ancient Interactions}

Interactions and mergers lead to the redistribution of material about a system. In search of traces left behind from past interactions, we co-added the $B$ - and $R$-band wide-field images of HCG 7 (presented in Section 3.3) and smoothed the resulting image with a $15 \times 15$ pixel boxcar. These two bands provide a good tracer of star formation, as they cover young stars and $\mathrm{H} \alpha$ emission, respectively; the $R$ band also covers light from old stars. We present the smoothed, co-added image at high contrast in Figure 9.

At first glance, there is no compelling evidence of major interactions among the four member galaxies; $C$ shows no signs of disturbance. However, we do find faint stellar features in the system, all related to $B$ : a streamer to its north, seemingly connecting it to the compact galaxy $E$, some $30 \mathrm{kpc}$ in projection. Based on its marginal detection in the $B$ band alone, we propose that star formation along this stream has long been extinguished. We find the color of this feature to be approximately $(B-R)=$ 1.1 , indicative of an old age. It compares to a BC03 SSP of age $\sim 2$ Gyr. This is an uncertain measurement, due to the marginal detection in the $B$ band, thus we consider this age a lower limit: if the stars formed in a tidal feature, then the passing of $E$ about $B$ occurred at that time. Otherwise, if the streamer consists of pre-existing stellar material, the youngest component of the mixed-age population has that age. In the following section, we consider the distribution if $\mathrm{H}_{\mathrm{I}}$ gas about HCG 7, in search of gaseous counterparts of the discovered streamers.

We also see the faint signature of an arc about $B$, extending to the east of the galaxy and possibly delineating the orbit of $E$ or $D$.

In turn, the central regions of $D$ present a symmetric light profile. Its spiral structure, however, is asymmetric, with the 
arm facing the side of $B$ appearing very diffuse. This may provide evidence of a past interaction between the two systems. Unfortunately, the stellar halos of the two galaxies are largely overlapping (Figure 9), therefore we cannot look for tidal features in the space between them.

Finally, $A$ : while its isophotes $A$ appear slightly irregular, this could be due to the imprint of its spiral structure at high inclination (this issue is revisited in Section 6). The space between $A$ and the $B / D$ complex may possibly feature another faint signature, although, again, this is enhanced by-if not entirely due to-the superposition of the two halos.

All of this information is telling of interactions. Given that collimated structures are short lived, especially in a complex tidal field, these interactions most likely happened in the past $\sim$ Gyr.

\subsection{The Gaseous Component of HCG 7}

We now turn our attention to the cold gas content of this galaxy group and analyze the available VLA $21 \mathrm{~cm} \mathrm{H}$ I observations. We find the gas to be contained entirely in the individual galaxies down to the $2.4 \times 10^{19}$ atom $\mathrm{cm}^{-2}$ sensitivity limit of our data set (derived in H10). This limit translates to a gas surface density of roughly $0.19 M_{\odot} \mathrm{pc}^{-2}$. This apparent absence of $\mathrm{HI}$ in the IGM implies a lack of major interactions between the four galaxies for at least one crossing time: $\tau_{\text {cross }}=R / \sigma \sim 60 \mathrm{kpc} / 100 \mathrm{~km} \mathrm{~s}^{-1} \sim 0.6$ Gyr. Note that the lack of three-dimensional velocity information and the small group membership make both parts of this fraction uncertain to a factor of 2 , so a $\tau_{\text {cross }} \sim 2 \mathrm{Gyr}$ is plausible.

This time frame is in accord with our understanding of dispersion timescales for tidal features: a suitable empirical upper limit of the ages of star clusters in such environments is $\sim 500 \mathrm{Myr}$. This value reflects the highest ages found for tidal clusters in previous studies (e.g., Schweizer \& Seitzer 1998; Trancho et al. 2007b). This empirical upper limit might be due to the fading of clusters with age, rather than their dispersal or destruction. Even in this case, our reasoning would still hold: HCG 7 clusters are subject to the same observational limits, therefore we can assume that $500 \mathrm{Myr}$ is the age of the oldest cluster we expect to be able to detect-be it due to fading or absence. Furthermore, Hibbard \& Yun (1999) detected an H I tidal structure in Arp 299 with an age of $\sim 750 \mathrm{Myr}$ with no optical counterpart. This implies that the gaseous component of tidal tails is more prevalent than the stars-as displayed, e.g.,in a plethora of multiple systems in the H I Rogues Gallery (Hibbard et al. 2001).

Additionally, the H I disks do not extend beyond their optical counterparts, an effect quite unusual outside the cores of galaxy clusters. This attribute may indicate that $\mathrm{H}$ I preprocessing has occurred within the galaxies, seemingly in the absence of major external influence. The exception here is $D$, which presents a slightly asymmetric $\mathrm{HI}$ distribution, reflected in its spiral structure. It is worth noting that the asymmetry is pointing toward $B$, however there are none of the tell-tale signs of a close encounter, such as a tidal bridge or young star clusters in the region. It is interesting to compare to the results of the Fabry-Pérot interferometry presented by Torres-Flores et al. (2009), who find evidence of a gaseous streamer directed at $B$. In addition, there is a clump of $\mathrm{H}$ I below the nucleus of $A$, cospatial with an $\mathrm{H} \alpha$ streamer detected in the Fabry-Pérot map (adapted in Figure 9). These two pieces of evidence strongly suggest an outflow from the nucleus of $A$, a very likely result of a starburst; we confirm this interpretation with our spectra in Section 6.1.

In Section 3.2, we derived the $\mathrm{H}$ I masses for $A / C / D$ as $\log$ $M_{\mathrm{HI}}=9.0,9.5,8.9 M_{\odot}$, with a null detection in the lenticular. H10 estimate an upper limit by properly accounting for the velocity width of an S0 galaxy and the rms error of the observations of $\log M_{\mathrm{HI}}^{B}<8.06 M_{\odot}$, a reasonable value for an evolved galaxy. These values are collected and contrasted with stellar masses and star formation characteristics in Table 2.

The resulting total $\mathrm{H}$ I mass of $M_{\mathrm{HI}}^{\text {tot }}=9.72 M_{\odot}$ is consistent with the single-dish measurement by Verdes-Montenegro et al. $(2001)^{19}$ and is approximately one-third of the average found in galaxy groups (Haynes \& Giovanelli 1984).

Naturally, hydrogen in galaxies is not only found in its neutral form, which is most likely inert in the star formation process (Kennicutt et al. 2007; Bigiel et al. 2008; Blanc et al. 2009; Krumholz et al. 2009). As stars form out of cool molecular gas, it is interesting to know the amount of $\mathrm{H}_{2}$ in each of the star-forming spirals. We draw these values from the study by Verdes-Montenegro et al. (1998), where $M_{\mathrm{H}_{2}}$ is derived through the amount of molecular $\mathrm{CO}$ (through observations of the $J=1 \rightarrow 0$ and $J=2 \rightarrow 1$ transition lines). The values for $A / C / D$ are $\log M_{\mathrm{H}_{2}}=9.7,<9.2,8.4$, where the value for $C$ is an upper limit. We note that the $\mathrm{CO}-$ to- $\mathrm{H}_{2}$ conversion factor $\left(X_{\mathrm{CO}}\right)$ is far from certain-derived values range between $\sim(2-3) \times 10^{20}$ with associated uncertainties of $\sim(0.7-1.5) \times 10^{20}$-therefore we treat these values with caution.

The above gas masses can now be used as a basis for an estimate of the timescale for depletion of the gas reservoir - both $\mathrm{H}_{\mathrm{I}}$ and $\mathrm{H}_{2}$. Following a rough assumption that (1) the current SFRs will be sustained until the depletion of all gas and (2) the $\mathrm{H}$ I reservoir is gradually being converted into $\mathrm{H}_{2}$ and thereafter into stars (with an eventual efficiency of $100 \%$ ), the timescale for the depletion of H I gas in HCG 7 is of the order of $1 \mathrm{Gyr}$ : $\log \tau_{\text {depletion }}=8.7,9.2,9.3 \mathrm{yr}$ for $A / C / D$. This provides another interesting comparison to the Leroy et al. (2008) sample, where the median value is $\log \tau_{\text {depletion }}=9.7 \mathrm{yr}$, i.e., a few Gyr longer. When considering the $\mathrm{H}_{2}$ values inferred by Verdes-Montenegro et al., the timescale for the $\mathrm{HI}+\mathrm{H}_{2}$ reservoir becomes an order of magnitude longer in the case of $A: \log \tau_{\text {depletion }}=9.2,9.4,9.4$ $\mathrm{yr}$ for $A / C / D$ (an upper limit in the case of $C$ ). This is due to the large $\mathrm{H}_{2}$-to-H I mass ratio of $A(\sim 5)$ that signifies that most of the gas is currently fueling star formation. We will revisit this intriguing find in Section 6. Unfortunately, given the uncertainty in the $X_{\mathrm{CO}}$ conversion factor, these values have a potentially large systematic uncertainty. We have omitted the contribution by He gas in the above calculation, as it is outweighed by the uncertainty in $X_{\mathrm{CO}}$.

\subsection{Galaxy B and the Origin of Group Lenticulars}

The formation of lenticular galaxies in clusters is still somewhat puzzling, therefore interpreting the origin of $B$ is of merit to understanding compact group evolution. In environments where major mergers are meant to be the norm, the evolution of a galaxy is likely to disrupt its disk structure-although recent modeling works by Combes (2008) and Brooks et al. (2009) have found disk re-growth a possibility in mergers where one of the parties is gas rich (also discussed in the context of "the Mice,"

\footnotetext{
19 Verdes-Montenegro et al. (2001) calculate $M_{\mathrm{H}}^{\text {tot }}=9.68 M_{\odot}$ using a single-dish measurement of the entire group; this small discrepancy is reasonable, considering that the interferometer is insensitive to large-scale $\mathrm{H} \mathrm{I}$ emission.
} 
NGC 4676, by Barnes 2002). This process does, however, require a timescale of a few Gyr and would most likely leave the imprint of a bimodality in the GC population, which will be treated in Section 5.

Validating such a scenario is beyond the reach of our diagnostic powers. Essentially, the galaxy shows no signs of massive cluster formation for at least the last $\sim 3$ Gyr. This implies that it entered a quiescent phase at that time, perhaps signaling the depletion of fuel. As a simple exercise, we can estimate the amount of stellar mass the galaxy has produced since that point in time. Assuming a constant SFR over the last $3 \mathrm{Gyr}$, equal to the rate currently observed, we derive a very low $M_{*}$ yield, suggesting that the galaxy produced $\sim 95 \%$ of its photometrically inferred $M_{*}$ before that time. By observation, isolated spirals do not deplete their gas reservoir in a Hubble time, so $B$ must have been aided in some way in processing the gas into stars. The presence of a disk structure and dynamics (i.e., the distinguishing factor between ellipticals and lenticulars) implies that the current state was not reached through a major merger.

In any case, the presence of an S0 type galaxy in a compact group that is apparently free of major interactions and mergers might suggest that lenticulars can indeed be sculpted by the conditions reigning in groups.

\section{THE GLOBULAR CLUSTER SYSTEM AS A TRACER OF PAST STAR FORMATION EPOCHS}

The study of old GCs expands our window for galactic archaeology to a much earlier era. Our HST imaging provides an exquisite opportunity to do so. In this section, we provide a full account of the GC system of HCG 7, constituting the first high-resolution study of globulars in compact group spirals/ lenticulars.

The color-color diagrams of Figure 7 show a significant population of stellar objects with colors consistent with that of old ( 10-12 Gyr) GCs; the majority are found around the lenticular $B$. We will treat the GC system of each galaxy separately, starting with the intertwined population of GCs around $B / D$.

\subsection{Galaxies $B$ and $D$}

From Figure 4, it is clear that the projected halos of the SB0 galaxy $B$ and the face-on spiral $D$ overlap, making a clean separation of their respective GC systems difficult. However, as $D$ is a spiral that has a much lower luminosity than $B$ $\left(M_{V}=-19.7\right.$ versus -21.0 for $\left.B\right)$, the number of GCCs expected in the halo of $D$ should be quite small. ${ }^{20}$ Thus, we will associate the GCCs surrounding galaxies $B$ and $D$ as being part of $B$, after first excising 11 GCCs that lie close to the center of $D$ - these objects may be affected by variable extinction internal to $D$. Of the $93 \mathrm{GCC}$ surrounding $B / D, 11$ near $D$ have been removed as noted above, leaving a total of 82 objects.

As only a fraction of the GC system of $B$ lies on our ACS fields, we derive the expected total number of GCs around $B$ by counting the GCs in a wedge (of angular extent 113 degrees) that extends to a distance of $30 \mathrm{kpc}$ from the center of $B$, yet lies wholly within the ACS field. From the observed number of $71 \pm 8$ objects that lie within this region, we predict a total of $227 \pm 27$ objects (assuming radial symmetry).

\footnotetext{
20 If we adopted $S_{N}=0.4$ for $D$ alone (e.g., Goudfrooij et al. 2003; Chandar
} et al. 2004), we would expect to detect $\sim 13$ GCCs in our ACS field.
Adopting a photometric completeness fraction $f=0.9 \pm 0.1$ to $M_{V}<-7.9$ (see discussion above) and correcting for the GCs below the magnitude cutoff, we derive a total number of $630 \pm 230 \mathrm{GCs}$ in $B$. For $M_{V}=-21.0$, this translates to a specific frequency (the number of GCs per unit galaxy luminosity) of $S_{N}=2.5 \pm 0.9$, in accord with global values of $S_{N}$ in the literature for S0 galaxies (both in the field and in the cluster environment; Ashman \& Zepf 1998; Kundu \& Whitmore 2001). The values of $S_{N}$ for S0 galaxies are (in general) higher (by a factor of $\sim 3$ ) than those for spiral galaxies, and this difference has been attributed to the fading (after the depletion of the original gas) of the stellar populations in spirals after a few Gyr (Aragón-Salamanca et al. 2006; Barr et al. 2007) and the consequent underdetection with respect to lenticulars.

Through the color distribution, we are also able to derive the metallicity distribution of the GCs in $B$. Figure 7 shows the $(B-I)_{0}$ colors of the candidates (transformed from the $B_{435}-I_{814}$ colors using the synthetic transformations from Sirianni et al 2005). The distribution does not show an obvious color bimodality common to many massive galaxies (e.g., Brodie \& Strader 2006; Peng et al. 2006). ${ }^{21}$ A single-Gaussian fit yields a peak $(B-I)_{0}=1.88 \pm 0.03$ (error in the mean value) and a dispersion of $0.23 \mathrm{mag}$. Under the assumption that all of the GCCs have ages similar to that of Milky Way GCs $(\sim 12 \mathrm{Gyr})$, we use the $(B-I)-[\mathrm{Fe} / \mathrm{H}]$ transformation from Harris et al. (2006):

$$
(B-I)_{0}=2.158+0.375[\mathrm{Fe} / \mathrm{H}]
$$

to convert these values to a peak $[\mathrm{Fe} / \mathrm{H}]=-0.74 \pm 0.08$ for the galaxy $B$ GCs. This value is very similar to the peak metallicities of GCs in other E/S0 galaxies, both in the field and in the cluster environment (e.g., Kundu \& Whitmore 2001; Peng et al. 2006).

Interestingly, recent modeling work by Muratov \& Gnedin (2010) interprets the bimodality of the GC color distribution to a few late mergers in the history of a given galaxy. If this is true, it lends strong support to our hypothesized lack of major mergers in HCG 7.

\subsection{Galaxy A}

The nearly edge-on $A$ shows a small number $(21 \pm 5)$ of GCCs, some projected onto its disk, others in the surrounding region. Adopting the same methodology and corrections as outlined above, this translates to a total expected number of $140 \pm 70$ within its halo. Assuming $M_{V}=-21.2$ gives rise to $S_{N}=0.4 \pm 0.2$. To investigate the color distribution of the GC system around $A$, we first extracted a subsample of 19 candidates located outside the center of the galaxy, so as to limit the contamination from any reddened younger clusters. The resulting $(B-I)_{0}$ color histogram in Figure 7 shows a wide range of colors, indicative of a wide range of metallicities. The mean color of the GCCs around $A$ is $(B-I)_{0}=1.83 \pm 0.05$, indicating a mean metallicity of $[\mathrm{Fe} / \mathrm{H}]=-0.88 \pm 0.13$ (assuming old cluster ages and the color-metallicity relation of Harris et al. 2006, used above). While there is little more that can be gleaned from this small sample, it is worth noting that the wide range of metallicities of the GCs in $A$ (the first time a metallicity distribution function has been constructed for

\footnotetext{
21 We confirmed the unimodal nature of the distribution through an attempt to fit the color distribution with a dual-Guassian fit using the KMM algorithm (Ashman et al. 1994); we find that such a fit is not significantly better than a single-Gaussian fit $(p=0.65)$.
} 
GCs in a spiral in the compact group environment) is similar to that observed in other spiral galaxies of similar luminosity (e.g., Barmby et al. 2000; Goudfrooij et al. 2003; Chandar et al. 2004).

\subsection{Galaxy $C$}

The orientation of face-on spiral $C$ impedes the extraction of GCs, as many clusters are expected to be heavily extinguished by internal dust, or simply lost in the variable background. Another caveat is the potential sample contamination from heavily reddened younger clusters; the lack of $U$-band information does not allow us the ability to clearly distinguish these possibilities. With this in mind, the total number of point sources with GClike colors that lie within $30 \mathrm{kpc}$ of $C$ is 29 . Assuming the entire GC system lies within our ACS fields, we derive a total GC population of $N_{T}=80 \pm 34$, i.e., a specific frequency of $S_{N}=0.3 \pm 0.2$. While highly uncertain, this number is consistent with expectations for late-type spiral galaxies (e.g., Chandar et al. 2004). As all of the GCCs are projected onto the disk of $C$, we are unable to extract a "clean" color/metallicity distribution.

\section{THE MULTI-WAVELEGTH VIEW: SPECTRA AND SEDs}

The SED of a galaxy provides valuable diagnostics of its overall properties. In this section, we look at the emission originating from the four group galaxies in the optical and near/ mid infrared $(0.4-24 \mu \mathrm{m})$. This is the combination of optical data from the SDSS, NIR $(J H K)$ data from 2MASS and mid-IR data from Spitzer (IRAC and MIPS; Section 3.1). The near- and mid-IR data are drawn from Johnson et al. (2007). We refer back to the optical spectra of galaxy nuclei to interpret the broadband emission and make use of the $\alpha_{\text {IRAC }}$ SED diagnostic discussed in Gallagher et al. (2008) and to a lesser extent in Section 3.1.

The SEDs, presented in Figure 8, are fit with appropriately chosen optical to far-IR galaxy templates from the GRASIL library (Silva et al. 1998). They reveal some very interesting aspects of the galaxies, all of which show an excess or dearth of emission in one of the observed bands, when compared to "normal" templates-i.e., ones representative of each galaxy's morphological type (as given in NED). To reconcile these discrepancies, we use appropriate "blends" of the individual emitting components in each GRASIL template: (1) star-forming molecular clouds and the young stars contained therein, (2) light from evolved stars and (3) diffuse gas. The three components are referred to as "MC," "starlight," and "cirrus" in Silva et al. (1998), the terminology that we hereforth adopt.

In this section, we also provide a closer examination of the central spectra obtained with the Hydra multi-object spectrograph, described in Section 3.4. We also compare our results to those of a recent spectroscopic campaign to identify active galactic nuclei (AGNs) in the full catalog of HCGs (Martínez et al. 2010).

\subsection{Galaxy A}

$A$ is listed as type Sa in NED; plotting the equivalent template produces a poor agreement with the observed mid-IR fluxes. Instead, we investigate the suitability of an Sb template. The $21 \mathrm{~cm}$ data reveal a low H I content in the HCG 7 galaxies, to the level of one-third the nominal value (Haynes \& Giovanelli 1984, see Section 4.6). Thus, we add only a third of the cirrus component to the template blend. Given the highly inclined line of sight toward the inner regions of $A$, we include only starlight extinguished by either dust or star-forming, gas-rich regions (i.e., no unextinguished starlight). Finally, we add the normal MC component. This template blend produces a very good match to the observed photometry.

Before dismissing the Sa designation, however, we perform a test for a possible AGN in galaxy $A$. We measure the strengths of emission lines $\mathrm{H} \beta$, [O III] $\lambda 5007, \mathrm{H} \alpha,[\mathrm{N} \mathrm{II}] \lambda 6584$, and $\left[\mathrm{S}_{\mathrm{II}}\right] \lambda \lambda 6717,6731$. We then compare the log ratios of $\left[\mathrm{O}{ }_{\mathrm{III}}\right] / \mathrm{H} \beta,[\mathrm{N} \mathrm{II}] / \mathrm{H} \alpha$, and $[\mathrm{S} \mathrm{II}] / \mathrm{H} \alpha$ to the values in Kewley et al. (2006, we refer to their Figure 4) to identify the presence of an AGN. Both the $\log ([\mathrm{N} \mathrm{II}] / \mathrm{H} \alpha)$ and $\log ([\mathrm{O} \mathrm{III}] / \mathrm{H} \beta)$ fall in the interface between $\mathrm{H}$ II nuclei and AGN. The likelihood of $A$ hosting an AGN is lessened by the value of the $\log ([\mathrm{S} \mathrm{II}] / \mathrm{H} \alpha)$ that places it clearly in the area occupied by H II nuclei. We can therefore attribute the high star formation activity in $A$ to a central starburst. The Martínez et al. (2010) study classifies $A$ as a "transition object" (TO), one that hosts an AGN and a circumnuclear starburst. This scenario is consistent with the mid-IR morphology of the galaxy disk, which shows bright central emission across the spectrum and $24 \mu \mathrm{m}$ emission in a limited halo. We also see a circumnuclear ring of stellar photospheric $(3.5 \mu \mathrm{m})$ emission (also detected as a faint region in the UV), which we interpret as low or even null star formation. This region is also devoid of gas down to our detection limit, indicating that star formation has slowed down or ceased around the nucleus either because the gas was spent in making stars, or that it was swept inward, perhaps owing to the presence of the bar.

With this information in mind, we construct a GRASIL Sa template with an MC (star formation) component boosted by five times the nominal Sa rate. In order to account for the excess dust produced in a starburst, we only include extinguished starlight. Finally, we maintain the cirrus emission to a third of the template value. This recipe does not provide a better fit to the data and is equivalent to the $\mathrm{Sb}$ template plotted in Figure 8. However, while the high inclination does not allow us to discern between these two distinct scenarios, the disk morphology does support currently strong central activity.

\subsection{Galaxy $B$}

$B$ matches the GRASIL 13 Gyr old elliptical galaxy template extremely well, except in the $24 \mu \mathrm{m}$ band, where it shows excess emission. In order to reconcile this effect, we compare it to a younger model, a distinct possibility for this lenticular galaxy. The presence of old globulars in this galaxy (Section 5) marks star formation activity at least $\gtrsim 5$ Gyr ago. Beyond this point, the evolution of GC BVI colors is very slow, therefore we cannot certainly distinguish the ages of GCs between 5 and 13 Gyr (as discussed above). The best agreement in the $24 \mu \mathrm{m}$ emission is offered by an 8 Gyr old elliptical template, although an age of 5 Gyr is not ruled out based on the SED.

\subsection{Galaxy $C$}

$C$ and $D$ both display a dearth of $24 \mu \mathrm{m}$ emission. The spectrum of $C$ is not dominated by blue continuum, as one would expect for the nucleus of an Sc galaxy. Instead, the overall shape is more reminiscent of an Sb-type. There is booming $\mathrm{H} \alpha$ emission, with an equivalent width (EW) of $-29 \AA$, however, the emission-line diagnostics do not show evidence of an intense ionization continuum. M10 classify the nucleus of $B$ as star forming, in tune with our analysis.

The reduced $24 \mu \mathrm{m}$ emission is reconciled by adopting an Sbtype template with low cirrus emission; this is the one shown in Figure 8. 


\subsection{Galaxy D}

This galaxy presents a very interesting " $E+A$ " central spectrum. Adopting for the moment its NED designation as Sc, one would expect to see hydrogen in strong emission lines, a flat spectral shape, and plenty of emission features below $\lambda 3700$. Instead, the spectrum of $D$ features moderate $\mathrm{H} \alpha$ emission and $\mathrm{H} \beta$ mostly in absorption, with only a weak emission feature. The upper series Balmer lines are seen in pure absorption, all the way to $\mathrm{H} \epsilon$, evident to the side of a weak $\mathrm{Ca}$ II $\mathrm{K}$ interstellar absorption line. The dominance of the stellar $\mathrm{H} \epsilon$ line over $\mathrm{Ca}$ II $\mathrm{H}$ suggests a low interstellar medium (ISM) content of the region covered in the spectrum, if not the entire galaxy. This is reflected in the reduced $24 \mu \mathrm{m}$ emission, which also originates from interstellar material, namely, medium-size dust grains in the case of $24 \mu \mathrm{m}$.

"E+A" spectra normally arise from a relatively recent and abrupt cessation of star formation activity in a previously starforming galaxy. What little star formation persists produces the mission component, which becomes embedded in the deep absorption lines of the recently formed stellar population and the underlying old stars.

We investigate the spectrum in this light through a model fit. We used the "penalized pixel fitting method" (Cappellari \& Emsellem 2004) to fit around the emission component and construct a template of the underlying continuum and absorption lines, as shown in the bottom panels of Figure 10. We then subtracted this template from the observed spectrum and measured the EWs of the $\mathrm{H} \delta$ in absorption and $\mathrm{H} \alpha$ in emission as $5.23 \pm 0.22 \AA$ and $-5.58 \pm 0.03 \AA$, respectively. These lines are not as wide as the archetypal post-burst galaxies selected by Zabludoff et al. (1996), implying that the recent epoch of star-forming activity was not quite as strong as a starburst.

Given the above, as well as the low gas and ISM content of the galaxy and its weak, asymmetric spiral structure, we suggest that this system is in a transitory stage between spiral and lenticular. At the current SFR this transition would take $\log \tau \sim 9.2-9.4 \mathrm{yr}$ (Section 4.6); this is an upper limit, as another interactiontriggered starburst would shorten this timescale.

The disagreement between template and observation in the optical regime is due to the previous misclassification of this galaxy as SB0: SDSS photometry is conducted in a manner consistent with morphological type, meaning that any emission from the (faint) spiral arms of this galaxy would have been omitted.

\section{IMPLICATIONS FOR THE EVOLUTION OF HCG 7}

\subsection{The Effect of Environment on the Evolution of HCG 7}

In Section 4.6, we discussed the $\mathrm{H}$ I distribution about the group. The VLA data do not detect an IGM in neutral gas and thus suggest that the HCG 7 galaxies have not undergone any major interactions recently, as such intense dynamical processes leave evidence for a few Gyr post facto. This was shown in the local universe by McConnachie et al. (2009, part of the PAndAS project) who recently presented the purely stellar tidal streams of M31 as the direct result of a 3 Gyr old encounter with M33. Naturally, with sources at distances of $\lesssim 1 \mathrm{Mpc}$, the PAndAS survey reaches much fainter detection limits compared to this study. Gaseous tidal features should, however, be detectable $\sim 1$ Gyr after the interaction in H I, as discussed in Section 4.6. The detection limit of the VLA data we use in this study is lower than the $10^{20} \mathrm{~cm}^{-2}$ level reached in a Jodrell Bank survey of the M81 group (Boyce et al. 2001), which uncovered previously undetected tidal features. In addition, the Rogues Gallery (Hibbard et al. 2001) showed very accentuated features at the $3 \times 10^{19} \mathrm{~cm}^{-2}$ level $\left(\simeq 0.8 M_{\odot} \mathrm{pc}^{-2}\right)$, a level easily accessible by our survey.

One additional caveat is the survivability of tidal features in relation to the environment: (e.g., Mihos 2001) showed that their lifetime is curtailed in crowded environments. The HST data and the wide-field imaging we analyzed in Section 4.5 address this issue: high SFR epochs are marked by massive clusters, the largest and brightest of which register as point sources. With the ability to confidently distinguish star clusters down to the 24 mag level $\left(M_{B}=-9 \mathrm{mag}\right)$, we can exclude major recent episodes of star formation in tidal debris.

The Chandra images treated by P. Tzanavaris et al. (2010, in preparation) show the four galaxies, but no IGM. This null detection of an X-ray IGM indicates that the missing $\mathrm{H}_{\mathrm{I}}$ is not concealed in a hot plasma phase that has been stripped from the galaxies during previous interactions.

At the same time, the current and past starbusts in $A / D$ are telling of active star formation activity across the group. In the absence of major interactions, AGNs, a hot IGM, and any other obvious energizing impulse, internal or external, we resort to the complex tidal field for an explanation of this activity. Simulations by Martig \& Bournaud (2008) find the intricate tidal field of groups and clusters to enhance bursts of mergerinduced star formation by a factor of up to six, although the exact physical mechanism behind this is not well understood. It might therefore be safe to assume a similar enhancement of star formation due to minor interactions between galaxies, since galaxies undergoing mergers and interactions are subject to similar gravitational processes; the result might not be the same, but the mechanism is similar. In this scenario, a grazing encounter can enhance the SFR compared to an identical system in isolation without the stripping and redistribution of gaseous material.

This is in accord with the study by Walker et al. (2010) who find evidence for rapid evolution in the compact group environment compared to clusters and the field. The mechanism is the depletion of the gas reservoir in a manner far quicker than field galaxies. However, like in the models by Martig \& Bournaud (2008), it is extremely difficult to pinpoint the physical process behind this relatively active star formation.

Ergo, we can conclude that this system is evolving quickly, under the influence of the tidal field; our observations suggest that a recent minor interactions have given rise to enhanced star formation, currently in $A$, and possibly recently in $D$.

\subsection{The Fossil Group: On the Path Toward a Dry Merger?}

The information derived in this work constrains the state of HCG 7 as a moderately star-forming, H I-deficient system. Whatever the mechanism driving its evolution, by combining the derived measurements we can make a prediction of the group's future. Considering the available reservoir of $\mathrm{H}$ I and $\mathrm{H}_{2}$, assuming that the galaxies are free to sustain their current rate of star formation (an upper limit in the case of $A$ ), they will convert the gas to stars within $\sim 2$ Gyr. This timescale, comparable to the crossing time (derived as $R / \sigma \lesssim 2 \mathrm{Gyr}$ in Section 4.6), may be too short for a series of major interactions to occur.

Following this hypothetical scenario, when the galaxies eventually converge to their common barycenter they would be devoid of gas and will therefore have no further material eligible for star formation. Such conditions would result in a 
"dry merger," whereby there is virtually no gas involved in the interaction (e.g., van Dokkum 2005). This would give rise to an elliptical galaxy, as per the regular end-state scenario for a galaxy group, however, without a bright X-ray halo, a feature that normally arises through the processing of intragroup gas. It must be stressed here, however, that the IGM is most likely present in compact groups, but due to their shallow potential, it was never heated to emitting temperatures and is not detectable. This means that a major fraction of the X-ray halo of the end state of a compact group will arise from this material. In this sense, the following consideration only applies to the evolution of compact groups, or arrangements of galaxies with a similar velocity dispersion.

We can test the validity of this hypothesis in terms of observations and theoretical predictions of the X-ray-to-blue luminosity ratio, $L_{X} / L_{B}$. Continuing on the dry merger toy model, let us assume that all gas will be internally processed, i.e., converted into stars, ahead of the final merger. In this case, the galaxy would have faint X-ray emission originating from its stellar halo. Given the stellar and gas masses in Table 2, we derive a total mass of $\log M_{\mathrm{TOT}}=10.70$, a mediumsized elliptical, considering that they reach masses in the order of $10^{13} M_{\odot}$. This value accounts for the contribution to the total mass by helium through the relation $M_{\mathrm{He}+\mathrm{H}_{\mathrm{I}}}=1.4 \times M_{\mathrm{HI}}$ (McGaugh et al. 2000). We can now estimate the blue luminosity through the $M / L$ of a BC03 model SSP of age $12 \mathrm{Gyr}^{22}$ : $M_{*} / L_{B} \simeq 2.0$, therefore $\log L_{B}=10.07$. Observations by O'Sullivan et al. (2007) provide a range of corresponding $\log L_{X}=39.5-41.5 \mathrm{erg} \mathrm{s}^{-1}$. Models by Cox et al. (2006) of mergers between disk galaxies result in the middle of that range, $\log L_{X} \simeq 41 \mathrm{erg} \mathrm{s}^{-1}$. A more conservative approach, where half the gas is used up in star formation and the rest released in the IGM through interactions leads to $M_{*}=10.64, \log L_{B}=10.35$, and $\log L_{X} \simeq 40.5 \mathrm{erg} \mathrm{s}^{-1}$ (from Cox et al. 2006) and a weak $\mathrm{X}$-ray IGM signature. Note that this emission is only due to the stellar X-ray halo, not the gas in the IGM. In either scenario, the $\mathrm{X}$-ray component is bound to be weak, because of the low mass of the fossil group. This is discussed in more detail in Gallagher et al. (2010) for the case of HCG 31.

In all, both scenarios are in excellent agreement with the two-pronged evolutionary sequence we presented in Section 1.1 and Figure 1, as well as the sequence proposed by VerdesMontenegro et al. (2001). In that arrangement, HCG 7 lies between Hickson groups 2 and 90: the former a collection of undisturbed, star-forming galaxies, the latter an apparent ongoing merger between two lenticular galaxies and a dusty spiral. It is important to note that this is a qualitative evolutionary sequence, as the masses of HCGs 2 and 90 are not in agreement with that of HCG 7. The arrangement of galaxies in the schematic of Figure 1 argues that the distribution of gas and arrangement of galaxies is crucial in defining the end state of a galaxy group.

The need for dry mergers in the history of the universe is exemplified by the large scatter of observed X-ray luminosities in truly isolated ellipticals (cf. recent observations presented by O'Sullivan et al. 2007; Memola et al. 2009). They are also important in populating the "red sequence" (Bell et al. 2004) and shaping the galaxy luminosity function. The possibility of systems like HCG 7 evolving in the way we suggest here would

\footnotetext{
22 This is clearly an oversimplification, as the hypothesized population would be a blend of stars of different ages. However, the small amount of gas left in the system at the present time would only give rise to a small young star population, thus the light would be dominated by old stars.
}

thus present an opportunity to "jump" back to $z \sim 1-2$ and observe X-ray weak ellipticals in the making.

\section{SUMMARY}

We have presented a multi-wavelength study of Hickson Compact Group 7. At first glance, this close grouping of four disk galaxies (three spirals, one lenticular) presents an overall seemingly undisturbed morphology: the individual members exhibit fairly regular disk/spiral structures and there are no evident tidal features in the optical, infrared, or radio data. Adding to this, the X-ray map of the group reveals no hot IGM. Finally, the age distributions of star cluster populations in the individual galaxies show no overdensities and hence no eras of pronouncedly elevated SFRs. At this point, one might conclude that HCG 7 consists of four neighbors that bizarrely remain "strangers," despite having spent billions of years in a volume that can hardly accommodate them comfortably.

Closer inspection reveals a different picture: spectra of the central regions of the galaxies uncover enhanced star formation activity in the past and present; at its lowest level, optical $B+R$ imaging shows faint stellar streamers connecting $D$ and the newly confirmed compact member $E$ to the evolved $B$; and the $\mathrm{H}$ I gas is not only heavily depleted-as confirmed by the UV-toIR SEDs of all galaxies-but also distributed in truncated disks that fall short of the galaxies' optical radii. In addition, we find evidence of fast gas depletion (compared to secularly evolving field galaxies), another indicator of past interactions, yet no trace of an H I or X-ray IGM. Our diagnostic tools are powerful enough to detect such features, therefore we conclude they are not there to be found. Finally, the unimodal color distribution of the GC population in galaxy $B$ might suggest a lack of major mergers in its history, according to the cosmological scale models of GC formation and evolution by (Muratov \& Gnedin 2010). This begs the question of why the galaxies are evolving in a manner similar to closely interacting systems.

While the physical mechanism behind this behavior is ultimately unclear, it is undoubtedly linked to the compact group environment. This is in tune with recent models of mergerinduced star formation in compact groups, where the SFR can be boosted by a factor of $\sim 6$, as compared to a merger occurring in the field (Martig \& Bournaud 2008).

We conclude this study by proposing a toy model for the future evolution and therefore the end state of this compact group. Our observations imply short depletion timescales for the little remaining gas. Assuming that the galaxies will not undergo major interactions or mergers in the next crossing time-as they have not during the last $\sim \tau_{\text {cross }}$ - there will be little gas in the eventual merger between the four galaxies. This paints the picture of the fossil group as an elliptical of average mass and with a faint (or no) X-ray component, originating solely from its stellar halo. This would differentiate it from the more studied evolutionary path that results in a gas-rich merger and a bright $\mathrm{X}$-ray halo that arises chiefly from IGM gas.

This image is in tune with the position of HCG 7 in the top prong of our proposed evolutionary sequence of compact groups. HCG 90, a representative end state for this sequence appears to be coming together with no detectable X-ray and H I components. This prospect may be extremely important in understanding the X-ray-to-optical luminosity ratio, $L_{X} / L_{B}$, observed at $z=0$.

We thank the anonymous referee for the valuable and positive input, which led to an improved manuscript. I.S.K. thanks 
Amanda Kepley for help with the interpretation of the radio data. We also thank Rodrigo Carrasco for useful discussions regarding the use of the Hydra iraf package. This work is based in part on observations made with the Spitzer Space Telescope, which is operated by the Jet Propulsion Laboratory, California Institute of Technology under a contract with NASA. Support for this work was provided by NASA through grant number HSTGO-10787.15-A from the Space Telescope Science Institute which is operated by AURA, Inc., under NASA contract NAS 5-26555, and the National Science and Engineering Council of Canada (S.C.G. \& K.F.). We acknowledge the financial support of NASA grant SAO SV4-74018 (PI: G. P. Garmire) which supports the Chandra ACIS team. P.R.D. acknowledges support from HST grant HST-GO-10787.07-A. Funding was provided by the National Science Foundation under award 0908984. K.E.J. gratefully acknowledges support for this work provided by NSF through CAREER award 0548103 and the David and Lucile Packard Foundation through a Packard Fellowship.

Facilities: HST, Spitzer, Blanco, VLA

\section{REFERENCES}

Anders, P., de Grijs, R., Fritze-v.Alvensleben, U., \& Bissantz, N. 2004, MNRAS, 347,17

Aragón-Salamanca, A., Bedregal, A. G., \& Merrifield, M. R. 2006, A\&A, 458, 101

Ashman, K. M., Bird, C. M., \& Zepf, S. E. 1994, AJ, 108, 2348

Ashman, K. M., \& Zepf, S. E. 1998, Globular Cluster Systems (Cambridge: Cambridge Univ. Press)

Barmby, P., Huchra, J. P., Brodie, J. P., Forbes, D. A., Schroder, L. L., \& Grillmair, C. J. 2000, AJ, 119, 727

Barmby, P., Kuntz, K. D., Huchra, J. P., \& Brodie, J. P. 2006, AJ, 132, 883

Barnes, J. E. 2002, MNRAS, 333, 481

Barr, J. M., Bedregal, A. G., Aragón-Salamanca, A., Merrifield, M. R., \& Bamford, S. P. 2007, A\&A, 470, 173

Bastian, N., Gieles, M., Efremov, Y. N., \& Lamers, H. J. G. L. M. 2005, A\&A, 443,79

Bastian, N., Trancho, G., Konstantopoulos, I. S., \& Miller, B. W. 2009, ApJ, 701, 607

Bell, E. F., et al. 2004, ApJ, 608, 752

Bergvall, N., Laurikainen, E., \& Aalto, S. 2003, A\&A, 405, 31

Bigiel, F., Leroy, A., Walter, F., Brinks, E., de Blok, W. J. G., Madore, B., \& Thornley, M. D. 2008, AJ, 136, 2846

Binggeli, B., Tammann, G. A., \& Sandage, A. 1987, AJ, 94, 251

Blanc, G. A., Heiderman, A., Gebhardt, K., Evans, N. J., \& Adams, J. 2009, ApJ, 704, 842

Borthakur, S., Yun, M. S., \& Verdes-Montenegro, L. 2010, ApJ, 710, 385

Boyce, P. J., et al. 2001, ApJ, 560, L127

Brodie, J. P., \& Strader, J. 2006, ARA\&A, 44, 193

Brooks, A. M., Governato, F., Quinn, T., Brook, C. B., \& Wadsley, J. 2009, ApJ, 694, 396

Bruzual, G., \& Charlot, S. 2003, MNRAS, 344, 1000

Cappellari, M., \& Emsellem, E. 2004, PASP, 116, 138

Chandar, R., Whitmore, B., \& Lee, M. G. 2004, ApJ, 611, 220

Combes, F. 2008, arXiv:0811.0153

Cox, T. J., Matteo, T. D., Hernquist, L., Hopkins, P. F., Robertson, B., \& Springel, V. 2006, ApJ, 643, 692

de Vaucouleurs, G., de Vaucouleurs, A., Corwin, H. G., Jr., Buta, R. J., Paturel, G., \& Fouque, P. 1991, Third Reference Catalogue of Bright Galaxies, Vols. 1-3, XII (Berlin: Springer), 2069

Efremov, Y. N., Ivanov, G. R., \& Nikolov, N. S. 1986, in IAU Symp. 116, Luminous Stars and Associations in Galaxies, ed. C. W. H. de Loore, A. J. Willis, \& P. Laskarides (Cambridge: Cambridge Univ. Press), 389

Eke, V. R., et al. 2004, MNRAS, 348, 866

Ellison, S. L., Simard, L., Cowan, N. B., Baldry, I. K., Patton, D. R., \& McConnachie, A. W. 2009, MNRAS, 396, 1257

Elmegreen, B. G., \& Elmegreen, D. M. 1983, MNRAS, 203, 31

Elmegreen, D. M., \& Salzer, J. J. 1999, AJ, 117, 764

Fruchter, A., Sosey, M., Hack, W., Dressel, L., Kockemoev, A. M., Mack, J., Mutchler, M., \& Pirzkal, N. 2009, The MultiDrizzle Handbook (Baltimore, MD: Space Telescope Science Institute)

Gallagher, S. C., Charlton, J. C., Hunsberger, S. D., Zaritsky, D., \& Whitmore, B. C. 2001, AJ, 122, 163
Gallagher, S. C., Johnson, K. E., Hornschemeier, A. E., Charlton, J. C., \& Hibbard, J. E. 2008, ApJ, 673, 730

Gallagher, S. C., et al. 2010, AJ, 139, 545

Genzel, R., Tacconi, L. J., Rigopoulou, D., Lutz, D., \& Tecza, M. 2001, ApJ, 563,527

Goudfrooij, P., Strader, J., Brenneman, L., Kissler-Patig, M., Minniti, D., \& Edwin Huizinga, J. 2003, MNRAS, 343, 665

Harris, W. E. 1996, AJ, 112, 1487

Harris, W. E. 2001, in Saas-Fee Advanced Course 28: Star Clusters, ed. L. Labhardt \& B. Binggeli (Berlin: Springer-Verlag), 223

Harris, W. E., Whitmore, B. C., Karakla, D., Okoń, W., Baum, W. A., Hanes, D. A., \& Kavelaars, J. J. 2006, ApJ, 636, 90

Haynes, M. P., \& Giovanelli, R. 1984, AJ, 89, 758

Hibbard, J. E., \& van Gorkom, J. H. 1996, AJ, 111, 655

Hibbard, J. E., van Gorkom, J. H., Rupen, M. P., \& Schiminovich, D. 2001, in ASP Conf. Ser. 240, Gas and Galaxy Evolution, ed. J. E. Hibbard, M. Rupen, \& J. H. van Gorkom (San Francisco, CA: ASP), 657

Hibbard, J. E., \& Yun, M. S. 1999, AJ, 118, 162

Hickson, P. 1982, ApJ, 255, 382

Hickson, P., Kindl, E., \& Auman, J. R. 1989, ApJS, 70, 687

Hickson, P., Mendes de Oliveira, C., Huchra, J. P., \& Palumbo, G. G. 1992, ApJ, 399,353

Holtzman, J. A., et al. 1992, AJ, 103, 691

Huchtmeier, W. K. 1997, A\&A, 325, 473

Iglesias-Páramo, J., \& Vílchez, J. M. 1999, ApJ, 518, 94

Johnson, K. E., Hibbard, J. E., Gallagher, S. C., Charlton, J. C., Hornschemeier, A. E., Jarrett, T. H., \& Reines, A. E. 2007, AJ, 134, 1522

Kennicutt, R. C., Jr. 1998, in ASP Conf. Ser. 142, The Stellar Initial Mass Function, ed. G. Gilmore \& D. Howell (38th Herstmonceux Conference; San Francisco, CA: ASP), 1

Kennicutt, R. C., Jr., et al. 2003, PASP, 115, 928

Kennicutt, R. C., Jr., et al. 2007, ApJ, 671, 333

Kewley, L. J., Groves, B., Kauffmann, G., \& Heckman, T. 2006, MNRAS, 372, 961

Konstantopoulos, I. S., et al. 2009, Nature, 461, 66

Konstantopoulos, I. S., Bastian, N., Smith, L. J., Trancho, G., Westmoquette, M. S., \& Gallagher, J. S., III 2008, ApJ, 674, 846

Konstantopoulos, I. S., Bastian, N., Smith, L. J., Westmoquette, M. S., Trancho, G., \& Gallagher, J. S. 2010, ApJ, 701, 1015

Kormendy, J., \& Kennicutt, R. C., Jr. 2004, ARA\&A, 42, 603

Krumholz, M. R., McKee, C. F., \& Tumlinson, J. 2009, ApJ, 699, L850

Kundu, A., \& Whitmore, B. C. 2001, AJ, 122, 125

Lada, C. J., \& Lada, E. A. 2003, ARA\&A, 41, 57

Larsen, S. S. 2004, A\&A, 416, 537

Leitherer, C., et al. 1999, ApJS, 123, 3

Leroy, A. K., Walter, F., Brinks, E., Bigiel, F., de Blok, W. J. G., Madore, B., \& Thornley, M. D. 2008, AJ, 136, 2782

Lisenfeld, U., et al. 2007, A\&A, 462, 507

Martig, M., \& Bournaud, F. 2008, MNRAS, 385, L38

Martínez, M. A., Del Olmo, A., Coziol, R., \& Perea, J. 2010, AJ, 139, 1199

McConnachie, A. W., Bastian, N., Gieles, M., \& Lamers, H. J. G. L. M. 2010 in IAU Symp. 266, Star Cluster: Basic Galactic Building Blocks throughout, Time and Space (Cambridge: Cambridge Univ. Press), 433

McGaugh, S. S., Schombert, J. M., Bothun, G. D., \& de Blok, W. J. G. 2000, ApJ, 533, L99

Memola, E., Trinchieri, G., Wolter, A., Focardi, P., \& Kelm, B. 2009, A\&A, 497, 359

Mendes de Oliveira, C., \& Hickson, P. 1994, ApJ, 427, 684

Mihos, J. C. 2001, ApJ, 550, 94

Moles, M., del Olmo, A., Perea, J., Masegosa, J., Marquez, I., \& Costa, V. 1994, A\&A, 285, 404

Muratov, A. L., \& Gnedin, O. Y. 2010, ApJ, 718, 1266

Oestlin, G., Bergvall, N., \& Roennback, J. 1998, A\&A, 335, 85

Östlin, G., Zackrisson, E., Bergvall, N., \& Rönnback, J. 2003, A\&A, 408, 887

O'Sullivan, E., Sanderson, A. J. R., \& Ponman, T. J. 2007, MNRAS, 380, 1409

Palma, C., Zonak, S. G., Hunsberger, S. D., Charlton, J. C., Gallagher, S. C., Durrell, P. R., \& English, J. 2002, AJ, 124, 2425

Peng, E. W., et al. 2006, ApJ, 639, 95

Plana, H., Mendes de Oliveira, C., Amram, P., \& Boulesteix, J. 1998, AJ, 116, 2123

Rasmussen, J., Ponman, T. J., Verdes-Montenegro, L., Yun, M. S., \& Borthakur, S. 2008, MNRAS, 388, 1245

Rejkuba, M., Greggio, L., Harris, W. E., Harris, G. L. H., \& Peng, E. W. 2005, ApJ, 631, 262

Rhode, K. L., Zepf, S. E., Kundu, A., \& Larner, A. N. 2007, AJ, 134, 1403

Robin, A. C., Reylé, C., Derrière, S., \& Picaud, S. 2003, A\&A, 409, 523 
Roming, P. W. A., et al. 2005, Space Sci. Rev., 120, 95

Rubin, V. C., Hunter, D. A., \& Ford, W. K. J. 1991, ApJS, 76, 153

Rupen, M. P. 1999, in ASP Conf. Ser. 180, Synthesis Imaging in Radio Astronomy II, ed. G. B. Taylor, C. L. Carlli, \& R. A. Perley (San Francisco, CA: ASP), 229

Salzer, J. J., Jangren, A., Gronwall, C., Werk, J. K., Chomiuk, L. B., Caperton, K. A., Melbourne, J., \& McKinstry, K. 2005, AJ, 130, 2584

Sanders, D. B., \& Mirabel, I. F. 1996, ARA\&A, 34, 749

Scheepmaker, R. A., Haas, M. R., Gieles, M., Bastian, N., Larsen, S. S., \& Lamers, H. J. G. L. M. 2007, A\&A, 469, 925

Schlegel, D. J., Finkbeiner, D. P., \& Davis, M. 1998, ApJ, 500, 525

Schweizer, F., \& Seitzer, P. 1998, AJ, 116, 2206

Silva, L., Granato, G. L., Bressan, A., \& Danese, L. 1998, ApJ, 509, 103

Sirianni, M., et al. 2005, PASP, 117, 1049

Skrutskie, M. F., et al. 2006, AJ, 131, 1163

Solomon, P. M., Rivolo, A. R., Barrett, J., \& Yahil, A. 1987, ApJ, 319, 730

Spergel, D. N., et al. 2007, ApJS, 170, 377

Tago, E., Einasto, J., Saar, E., Tempel, E., Einasto, M., Vennik, J., \& Müller, V. 2008, A\&A, 479, 927

The, L. S., \& White, S. D. M. 1986, AJ, 92, 1248

Torres-Flores, S., Mendes de Oliveira, C., de Mello, D. F., Amram, P., Plana, H., Epinat, B., \& Iglesias-Páramo, J. 2009, A\&A, 507, 723

Trancho, G., Bastian, N., Miller, B. W., \& Schweizer, F. 2007a, ApJ, 664, 284

Trancho, G., Bastian, N., Schweizer, F., \& Miller, B. W. 2007b, ApJ, 658, 993

Tzanavaris, P., et al. 2010, ApJ, 716, 556 van Dokkum, P. G. 2001, PASP, 113, 1420

van Dokkum, P. G. 2005, AJ, 130, 2647

Verdes-Montenegro, L., Sulentic, J., Lisenfeld, U., Leon, S., Espada, D., Garcia, E., Sabater, J., \& Verley, S. 2005, A\&A, 436, 443

Verdes-Montenegro, L., Yun, M. S., Perea, J., del Olmo, A., \& Ho, P. T. P. 1998, ApJ, 497, 89

Verdes-Montenegro, L., Yun, M. S., Williams, B. A., Huchtmeier, W. K., Del Olmo, A., \& Perea, J. 2001, A\&A, 377, 812

Walker, L. M., Johnson, K. E., Gallagher, S. C., Hibbard, J. E., Hornschemeier, A. E., Charlton, J. C., \& Jarrett, T. H. 2009, arXiv:0908.2971

Walker, L. M., Johnson, K. E., Gallagher, S. C., Hibbard, J. E., Hornschemeier, A. E., Charlton, J. C., \& Jarrett, T. H. 2010, BAAS, 41, 237

White, P. M., Bothun, G., Guerrero, M. A., West, M. J., \& Barkhouse, W. A. 2003, ApJ, 585, 739

Whitmore, B. C., Zhang, Q., Leitherer, C., Fall, S. M., Schweizer, F., \& Miller, B. W. 1999, AJ, 118, 1551

Whitmore, B. C., et al. 2010, AJ, 140, 75

Williams, B. A., \& Rood, H. J. 1987, ApJS, 63, 265

Wilman, D. J., Oemler, A., Mulchaey, J. S., McGee, S. L., Balogh, M. L., \& Bower, R. G. 2009, ApJ, 692, 298

York, D. G., et al. 2000, AJ, 120, 1579

Zabludoff, A. I., \& Mulchaey, J. S. 1998, ApJ, 496, 39

Zabludoff, A. I., Zaritsky, D., Lin, H., Tucker, D., Hashimoto, Y., Shectman, S. A., Oemler, A., \& Kirshner, R. P. 1996, ApJ, 466, 104

Zhang, Q., \& Fall, S. M. 1999, ApJ, 527, L81 